Article

\title{
Effects on the Double Bounce Detection in Urban Areas Based on SAR Polarimetric Characteristics
}

\author{
José Manuel Delgado Blasco ${ }^{1,2, *}$, Magdalena Fitrzyk ${ }^{3}$, Jolanda Patruno ${ }^{1}$, \\ Antonio Miguel Ruiz-Armenteros ${ }^{2,4,5}$ and Mattia Marconcini ${ }^{6}$ \\ 1 RHEA Group, Via di Grotta Portella 6/8, 00044 Frascati, Italy; j.patruno@rheagroup.com \\ 2 Grupo de Investigación Microgeodesia Jaén (PAIDI RNM-282), Universidad de Jaén, \\ Campus Las Lagunillas s/n, 23071 Jaén, Spain; amruiz@ujaen.es \\ 3 RSAC c/o ESA-ESRIN, Largo Galileo Galilei 1, 00044 Frascati, Italy; Magdalena.Fitrzyk@esa.int \\ 4 Dpto. Ingeniería Cartográfica, Geodésica y Fotogrametría, Universidad de Jaén, EPSJ, \\ Campus Las Lagunillas s/n, Edif. A3, 23071 Jaén, Spain \\ 5 Centro de Estudios Avanzados en Ciencias de la Tierra, Energía y Medio Ambiente CEACTEMA, \\ Universidad de Jaén, Campus Las Lagunillas, s/n, 23071 Jaén, Spain \\ 6 German Aerospace Center (DLR), Oberpfaffenhofen, 82234 Wessling, Germany; mattia.marconcini@dlr.de \\ * Correspondence: j.delgado@rheagroup.com
}

Received: 18 February 2020; Accepted: 2 April 2020; Published: 7 April 2020

\begin{abstract}
Synthetic Aperture Radar (SAR) polarimetric datasets are widely used in the detection and classification of urban areas. Most methods used today are based on the decomposition of fully polarimetric SAR data, which allows for the extraction of physical information about the nature of the medium and the application of proper classification methods. According to the theory, the main and predominant backscattering mechanism for buildings is double bounce. However, when analyzing urban environments, the observed predominant backscatter may differ from theory depending on many aspects. In this paper, we analyze fully polarimetric ALOS PALSAR data for various cities located on different continents, proving that the theory does not hold for most cases. There are many factors that have an impact on the detected backscatter mechanism, and the theoretical principle of predominant double bounce in urban areas can be met only under specific conditions. These factors are, among others, the orientation of the buildings, the dimensions of the streets, the type of construction (i.e., numerous planes on the roof), etc. This paper also mentions the canonical example of San Francisco, widely analyzed in the literature, as a case showing the impact of building deorientation on double bounce scattering. This area of interest is also discussed in terms of the impact of SAR data resolution on the detection of specific backscatter mechanisms. The findings of this work are very useful for increasing the awareness of the utilization of classification approaches where only pixels with double bounce backscatter mechanisms are classified as urban areas. Moreover, the article lists factors that should be taken into consideration when performing urban area detection based only on polarimetric data and standard algorithms, such as street and building orientation, building heights, and structures.
\end{abstract}

Keywords: buildings; urban areas; radar polarimetry; full-polarimetric SAR; backscatter mechanisms; double bounce; Yamaguchi decomposition; ALOS PALSAR; world settlements footprint

\section{Introduction}

The classification and delineation of urban areas is still a challenging task in applied remote sensing. Over the last decades, there have been many studies on urban area detection using optical remote sensing imagery, as well as Synthetic Aperture Radar (SAR) data. The latter has many advantages 
due to the possibility of object detection even in the presence of clouds or heavy pollution. One of the features of SAR data, which can be useful in target detection and land cover classification, is the radar wave polarization, because the polarization information contained in the waves backscattered from a given medium is highly related to its geometrical structure, reflectivity, shape, orientation, and geophysical properties [1]. Fully polarimetric SAR data can be used to extract physical information about the nature of the medium from which the radar signal was backscattered. There are many approaches to the utilization of fully polarimetric data in urban areas recognition and classification. Most of them use polarimetric descriptors, such as the coherency matrix [T] or the covariance matrix, and apply polarimetric decomposition, which allows for the expression of radar backscatter from an object as a combination of scattering responses from a number of simpler objects with known backscatter characteristics (e.g., plane, sphere) or to specify the dominating scattering mechanisms.

As described in [2], there are many polarimetric decompositions, such as eigenvector-based incoherent decompositions, e.g. Cloude and Pottier [3], or model-based decompositions, e.g. Freeman-Durden [4] or Yamaguchi [5]. Applying model-based polarimetric decomposition (e.g., Freeman-Durden) on fully polarimetric data allows for the decomposition of the backscattered power of the signal into specific scattering mechanisms: single bounce, double bounce, and volume scattering, which can be assigned typically to surfaces, buildings, and vegetation, respectively (Figure 1) [1,5-7]. This approach, however, has an assumption of reflection symmetry, which sets a limitation for its applicability, especially in urban areas where rotated buildings (facades not parallel with respect to the satellite flight direction) produce strong cross-pol backscatter. This may lead the model-based decomposition to interpret urban areas as being dominated by volume scattering, which is typical for natural objects like vegetated areas [6].

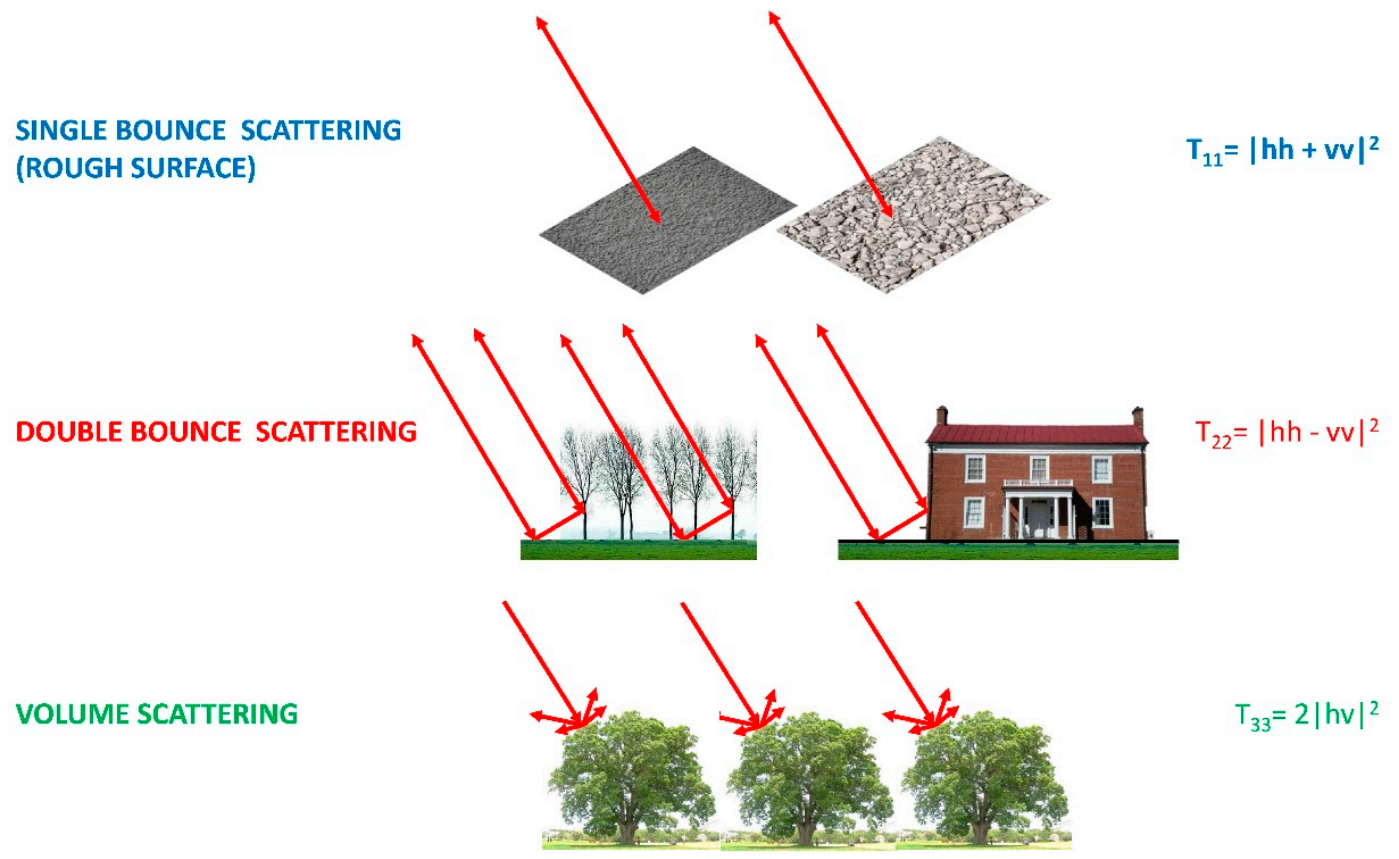

Figure 1. Main backscatter mechanisms obtained with the polarimetric coherence matrix [T3].

According to literature, the Yamaguchi four component decomposition [5,7] is the most suitable polarimetric decomposition for analyzing urban environments [2]. This decomposition is an extension of the three-component scattering power model by Freeman and Durden. It allows for not only the identification of the three aforementioned backscatter mechanisms but also the Helix backscatter mechanism, which appears in heterogeneous areas, for example, for man-made structures, and disappears for natural objects [5]. Although this decomposition can be used for identifying urban areas, there are still some challenges, such as, for example, target deorientation (or rotation), which could 
influence reliable target recognition. Some attempts have been carried out, such as [8], which partially improved the recognition of deoriented targets, but these improvements are still not integrated in polarimetric software such as POLSARpro toolbox [9].

One of the examples of urban area analysis using full-polarimetric datasets, often mentioned in the literature, is the city of San Francisco. Authors use various polarimetric decompositions [10-14] and classification methods [3,11,15-19] on several full-polarimetric datasets, for instance ALOS PALSAR, AIRSAR, and RADARSAT-2 to extract urban area and study the structure of the city. In our study we also had a look at the Pauli decomposition [1] of ALOS PALSAR full-polarimetric dataset for San Francisco and what could be noticed from this analysis is that the key and dominating backscatter mechanism within the city is double bounce, resulting from the scattering from the ground coupled with scattering from the faces of buildings (Figure 2). A is the Pauli Red Green Blue (RGB) false composite (Figure 2A), representing the combination of the three main scattering mechanisms: single (odd) bounce, double bounce, and volume scattering (assigned to blue, red, and green channels, respectively). These band assignments make urbanized areas appear in red and magenta as a combination of single (Figure 2B) and double bounce scattering (Figure 2C). This example also shows the overestimation of volume scattering for the areas with buildings rotated with respect to the satellite flight direction (Figure 2D), as will be explained in the following sections. Hence, the San Francisco case became a motivation to investigate if the same scattering mechanisms also dominate for other cities like Greater Cairo or New York.

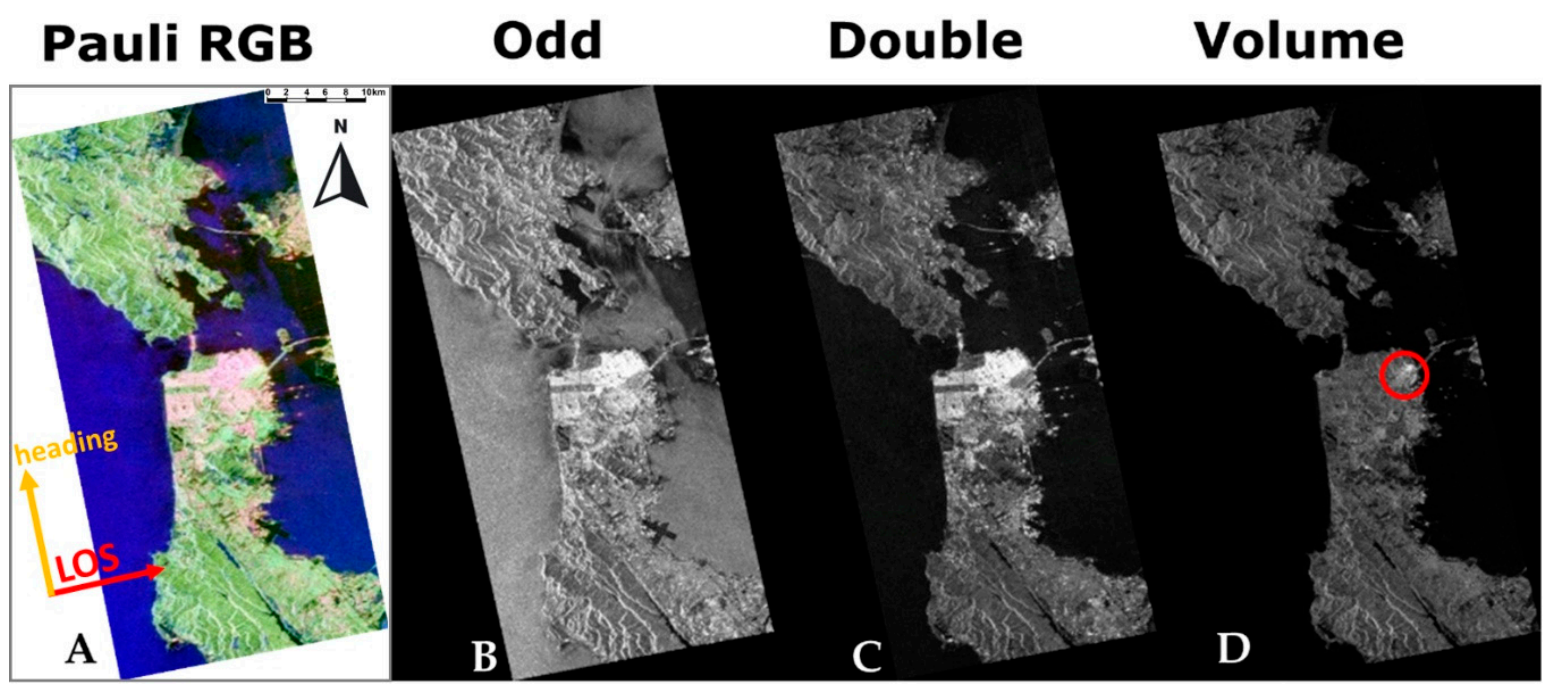

Figure 2. (A) Pauli RBG, (B) Single (Odd) bounce, (C) double bounce, and (D) volume scattering mechanisms over San Francisco using the ALOS PALSAR dataset. Deoriented buildings are located within the red circle in (D).

The goal of this paper is to investigate the backscatter response of urban areas, in order to understand whether the theoretical domination of the double bounce backscatter response from buildings is true for the majority of urban areas, in various urban agglomerations, and to identify factors, besides the target deorientation, which may affect the backscatter response over urban areas and lead to ambiguous results of urban area detection.

\section{Study Areas}

In this paper, we present seven different study areas. Each area of interest (AOI) represents urban agglomeration areas or megacities located on four different continents (Figure 3). The choice of these AOIs was the result of a literature study on the characteristics of urban agglomerations in different parts of the world. In [20], Loibl et al. presented a summary of various spatial patterns that can be observed in the structure of European, Asian, Latin American, and North American cities, with the 
emphasis on the shape of the urban area and the directions of its expansion. Specific characteristics of urban agglomerations, also depending on their geographical location, led us to choose the following cities: Beijing and Shanghai from Asia, Milan from Europe, New York, San Francisco, and Los Angeles from North America, and Greater Cairo from Africa. A detailed description of each AOI is presented in the following subsections. Each city was analyzed within their known extent obtained from the World Settlement Footprint Evolution (WSF) [21,22].

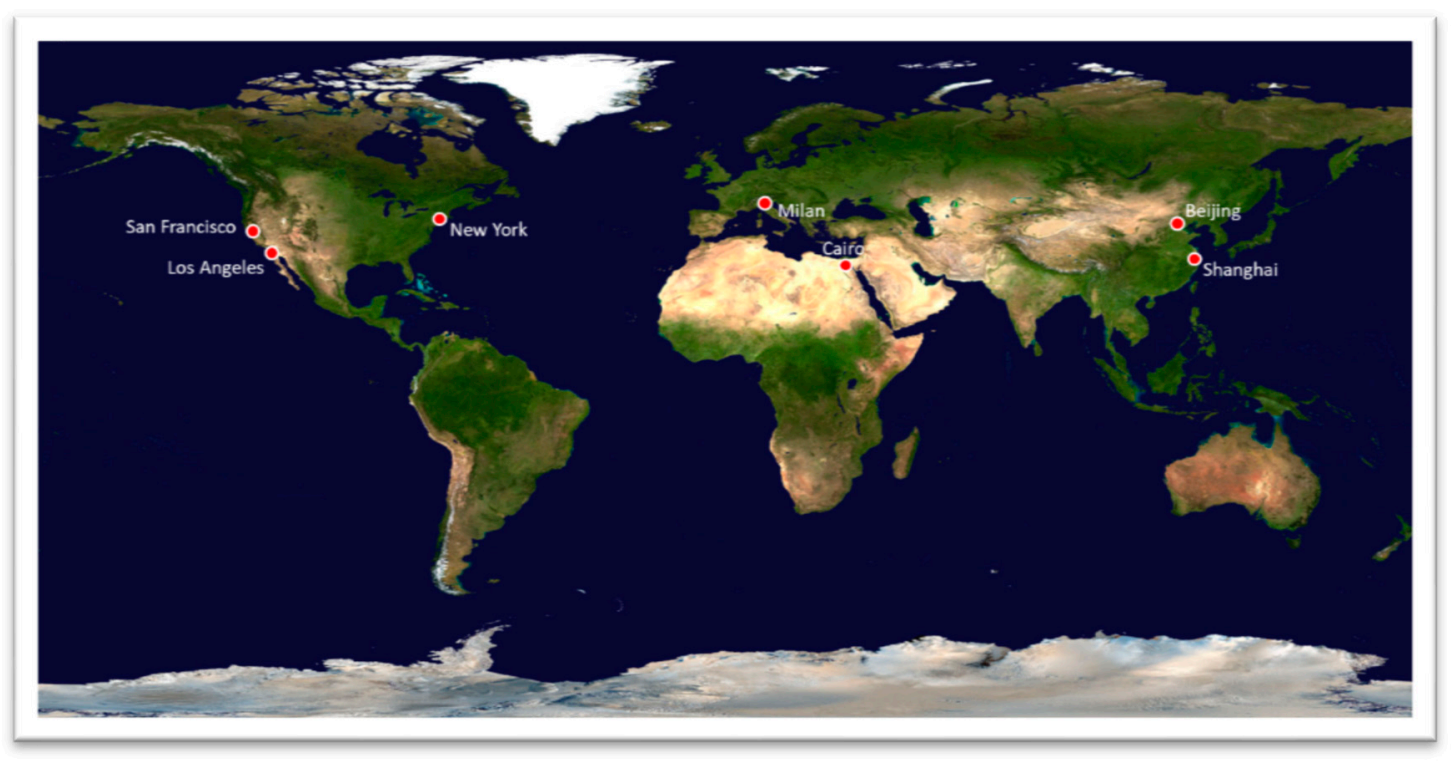

Figure 3. Location of the selected cities shown over the blue marble picture (source: NASA).

\subsection{Beijing}

The city of Beijing (Figure 4) is located at the northwestern edge of the North China Plain. The total area of Beijing is 16,808 $\mathrm{km}^{2}$, with population exceeding 21 million inhabitants, of which more than $80 \%$ live in urban areas [23]. The urban structure of Beijing traces back to the early fifteenth century and the foundation of the Forbidden City and the Temple of Heaven-the governmental and spiritual centers of old Beijing [20]. The old Beijing was planned as a system of circular rings centered around the Forbidden City. Since the 1960s, following increasing urbanization, new rings were added, with the structure of urban metropolis [20]. Currently, in the urban spatial structure of Beijing, we can distinguish traditional housing, historical post-imperial palaces, as well as modern skyscrapers, the majority of which are located in the eastern part of the metropolis. The streets of the inner city are well organized, with wide main streets, tall buildings located along them, and lower buildings on secondary streets [24]. 


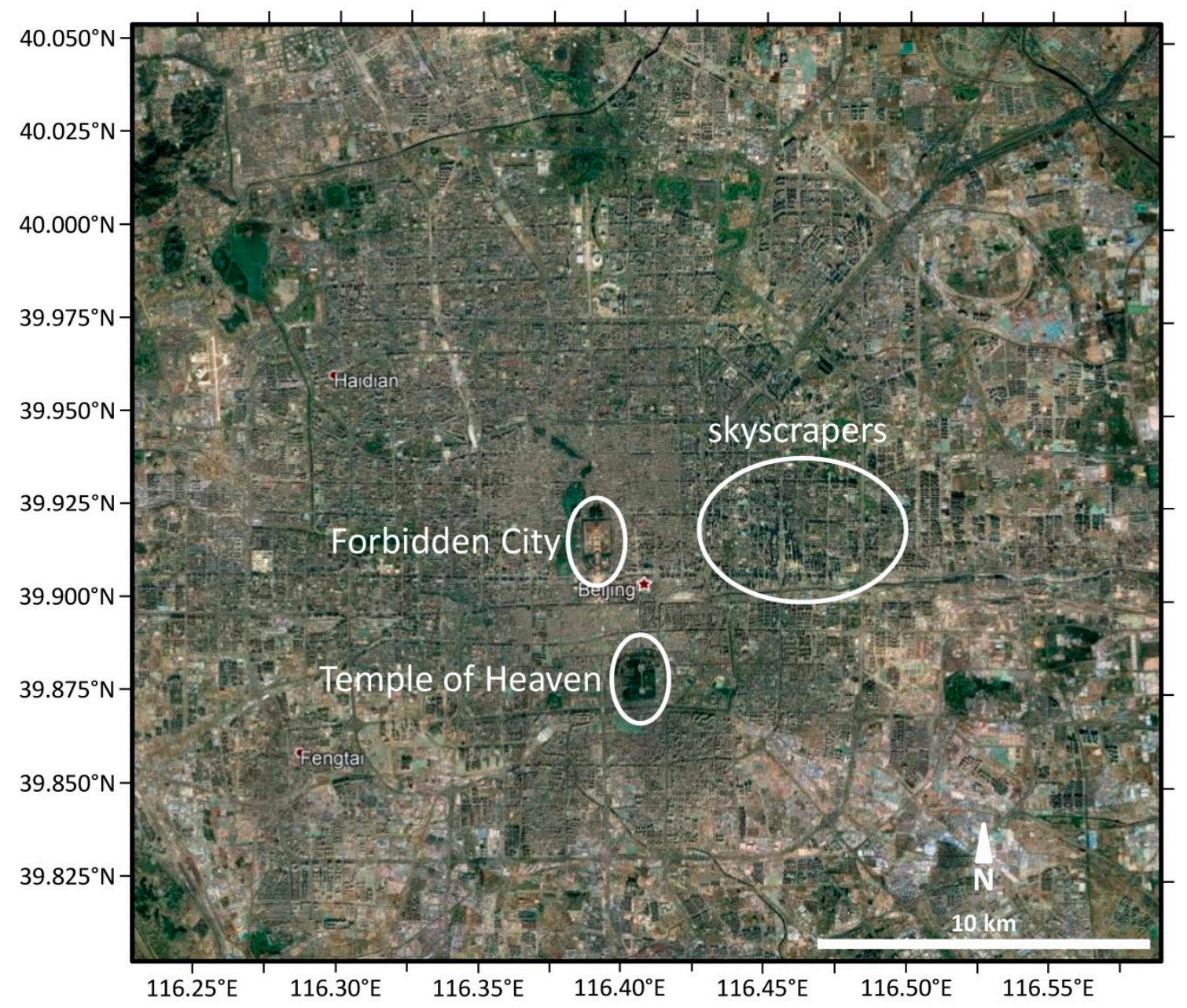

Figure 4. Map of Beijing (background image: Google).

\subsection{Shanghai}

Shanghai (Figure 5), one of the China's megacities, is similar to Beijing. With a population of more than 24 million inhabitants, it is the most populous urban area in China. The city is located on the east coast of China, on the southern estuary of the Yangtze River. Due to its location and geographic conditions the morphology of Shanghai can be defined as plain city (plain areas around the main city) with circular urban sprawl pattern affected by natural setting. The city can be divided by the Huangpu River into two main parts called Puxi and Pudong, the latter being the $h$ of the famous business district. The streets in Shanghai follow the Chinese traditional pattern of street network in which roads comply with the four cardinal orientations; however, it lacks a well-formed grid system. This is especially visible in areas developed later which seem more random. The specific street pattern of Shanghai can be related mainly to the natural/geographical conditions, but also to some economic factors such as the subdivision of foreign concessions and the assignment of their development to different private owners. 


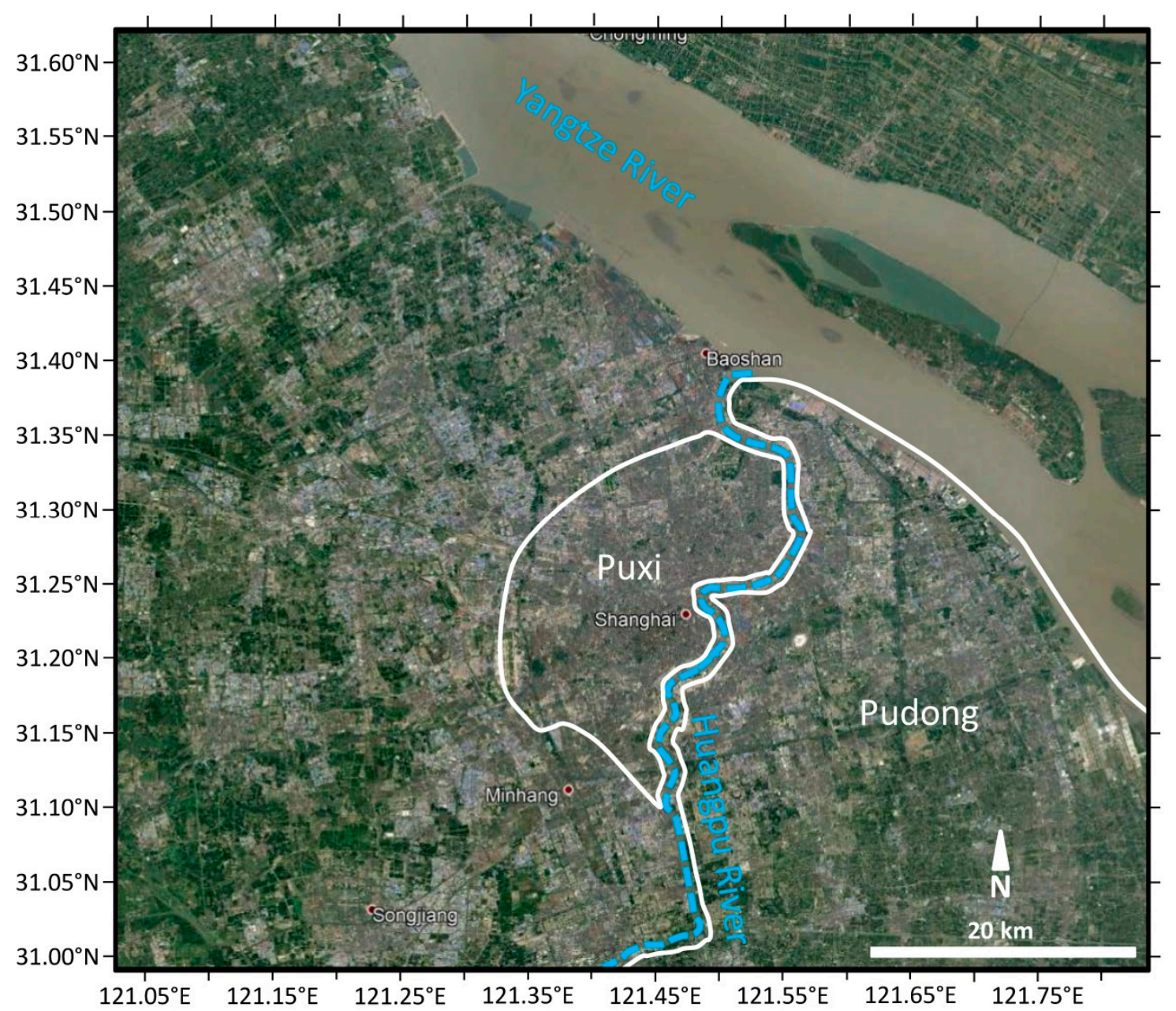

Figure 5. Map of Shanghai (background image: Google).

\subsection{Milan}

The city of Milan (Figure 6) is located in the north of Italy and is the largest city of the Lombardy region with its urban extent of $181.8 \mathrm{~km}^{2}$, and the second Italian city by total population. The population of the city itself is around 1.4 million, whereas the whole metropolitan area has more than 3 million inhabitants. The city is divided into nine municipalities, of which the first zone, called "Municipio 1 ", corresponds to the historical center. Most of the buildings in the strict center were constructed in the 14th-15th century; however, some of the ancient Roman remains can be also spotted. Most of the palaces existing in the city can be dated back to the 18th-20th century. Zone 2, called Porta Nuova, is the main business district in Milan and the home of the highest skyscrapers, e.g., Unicredit Tower. The other area that includes the tallest buildings is zone 8 - CityLife. 


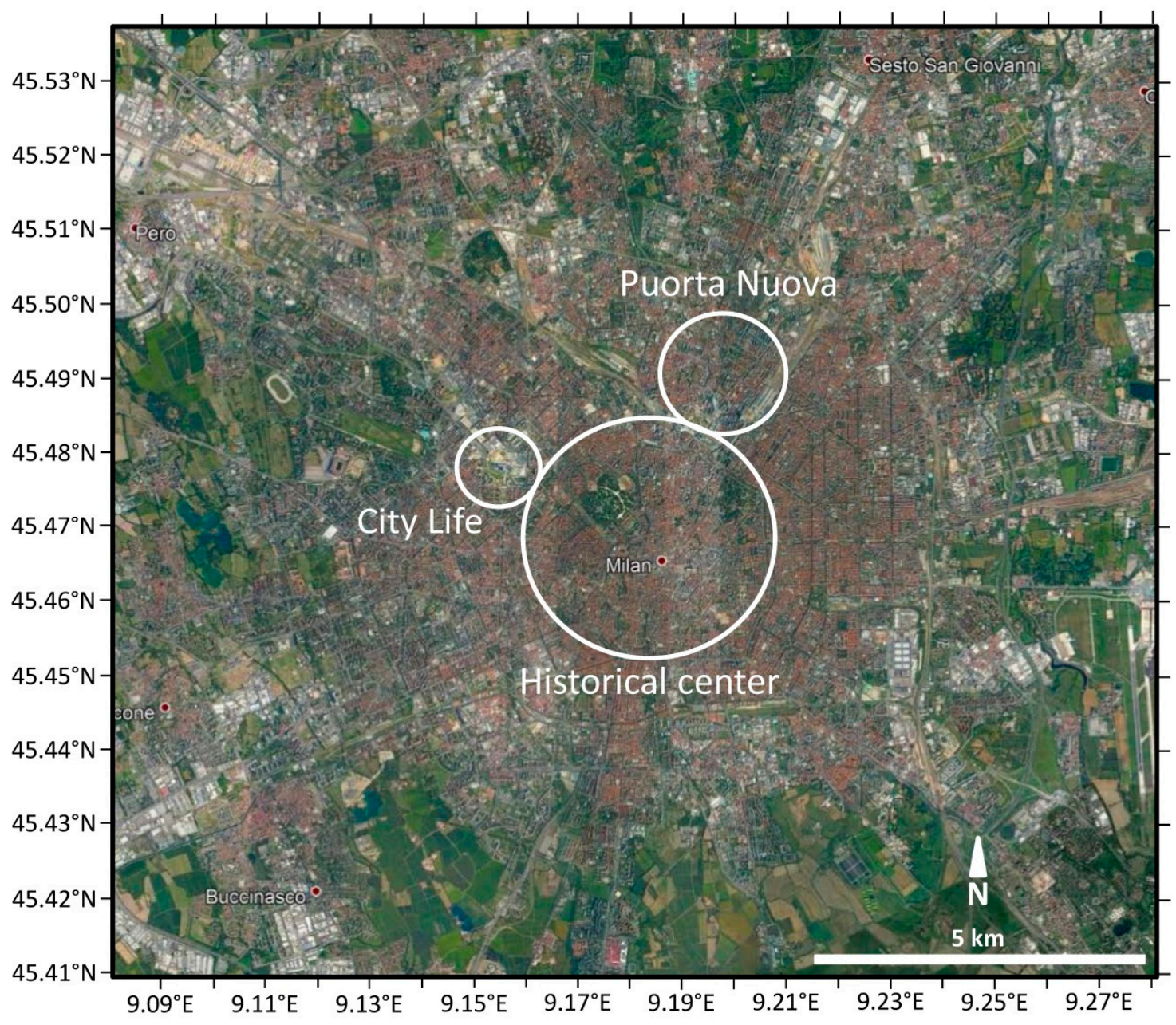

Figure 6. Map of Milan (background image: Google).

\subsection{New York}

The city of New York (Figure 7) is located at the southern tip of the state of New York, on one of the world's largest natural harbors. It covers a land area of about $780 \mathrm{~km}^{2}$ and is the most densely populated major city in the United States. The city plan (for its central part-Manhattan) was based on the Commissioners' Plan of 1811, which imposed the design of streets in a rectangular grid. The huge urban park in Manhattan-Central Park-was not a part of this plan since it was not foreseen until the 1850s. Moreover, lower Manhattan does not follow the grid pattern as it has its origins earlier in the $17^{\text {th }}$ century and it was influenced by Dutch settlers who called it the New Amsterdam. Currently, Manhattan is the center where the main skyscrapers are located. The spatial structure of other boroughs, like Brooklyn or Queens, follow a more irregular grid pattern with a lower skyline dominated by residential buildings. 


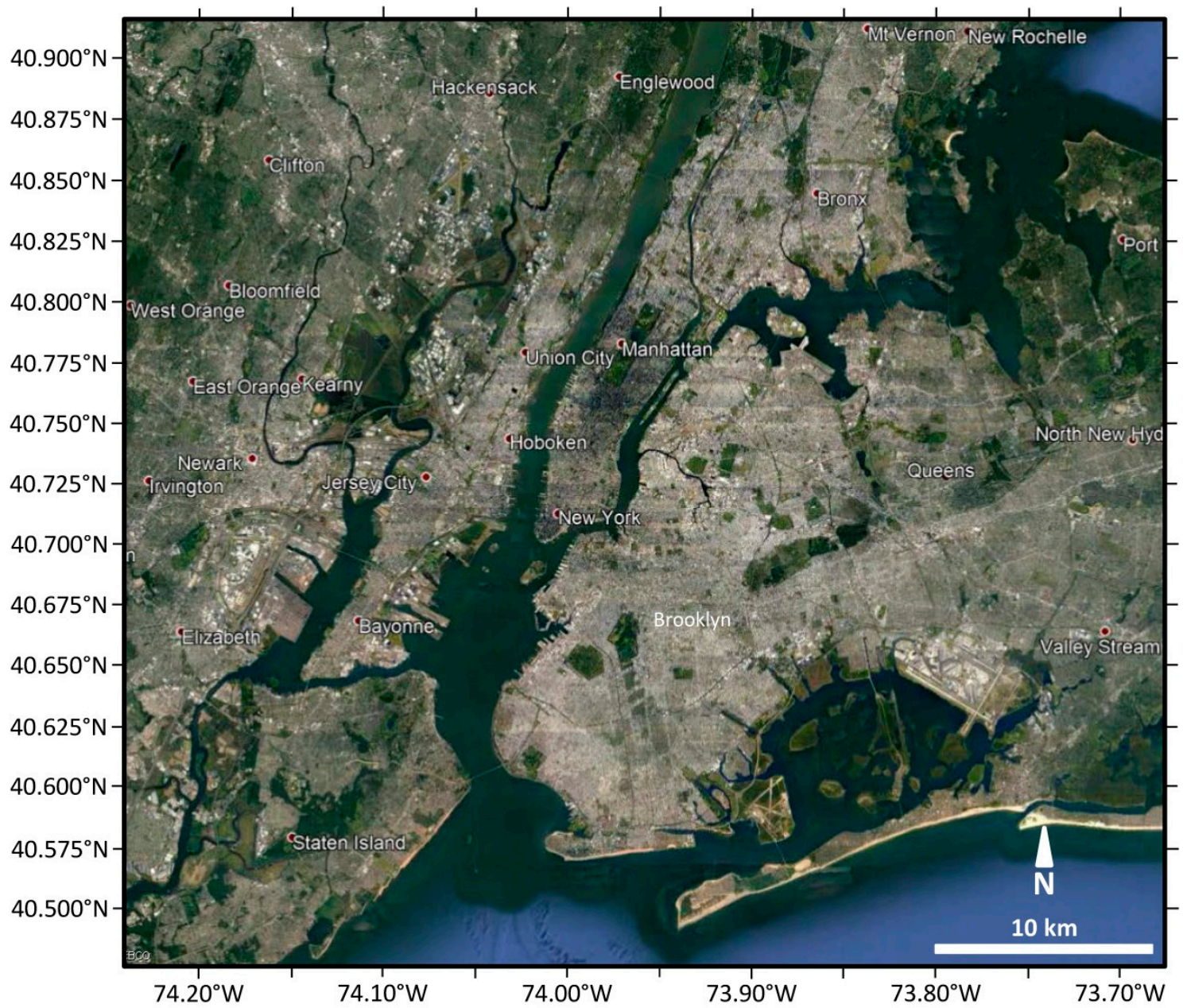

Figure 7. Map of New York (background image: Google).

\subsection{San Francisco}

The city of San Francisco (Figure 8) is located in North America, in Northern California. It covers an area of about $120 \mathrm{~km}^{2}$, most of which is located in the San Francisco Bay Area. The city was founded in the 1770s and grew rapidly in the following century, becoming the largest city on the West Coast. It was originally laid out on a grid pattern imposed on hills. In 1906, the city was destroyed by an earthquake, after which it was re-built. San Francisco is a very well-planned city, extremely dense, and with very minimal area for expansion. In the city structure, we can distinguish four main zones: an office district with high-rise office and corporate buildings, a retail district with shopping centers, a general commercial district with hotels and restaurants, and a sport/recreation district. The largest recreation area-Golden Gate Park, located in the western part of the city-covers an area of approximately $4.12 \mathrm{~km}^{2}$. The highest skyscrapers are located in the financial district in the NE of the city. The south of the market district, located south from the financial district, is very characteristic for its squinted grid of streets, not following the orientation of grids in the surrounding districts. 


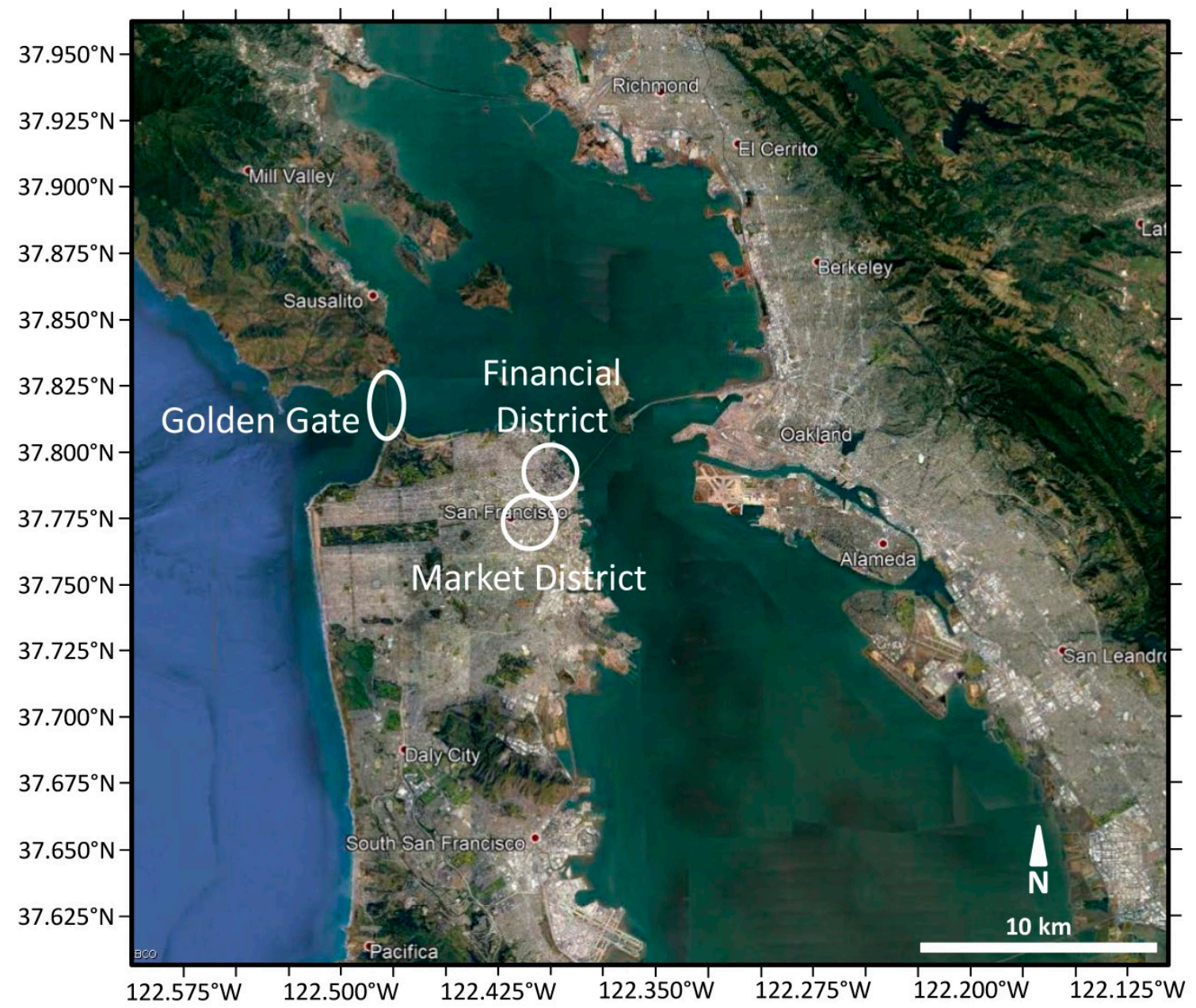

Figure 8. Map of San Francisco (background image: Google).

\subsection{Los Angeles}

Los Angeles (Figure 9) is located in North America, which is now one of the most urbanized areas of the world, with about $82 \%$ of people living in urban areas [20]. It lies in a basin of Southern California, adjacent to the Pacific Ocean. The city covers an area of approximately $1200 \mathrm{~km}^{2}$ and is the most populous county in the United States. The urban pattern of the city follows the Land Ordinance of 1784, according to which the land would be divided into regular squares and would follow the principles of orthogonal planning with the main streets following the cardinal directions [25]. In the panorama of the city, we can distinguish various zones, including residential areas, commercial space, and open space/public zones. The area with skyscrapers is centered at the tallest building in the city-Wilshire Grand Tower located in the NW part of the agglomeration. 


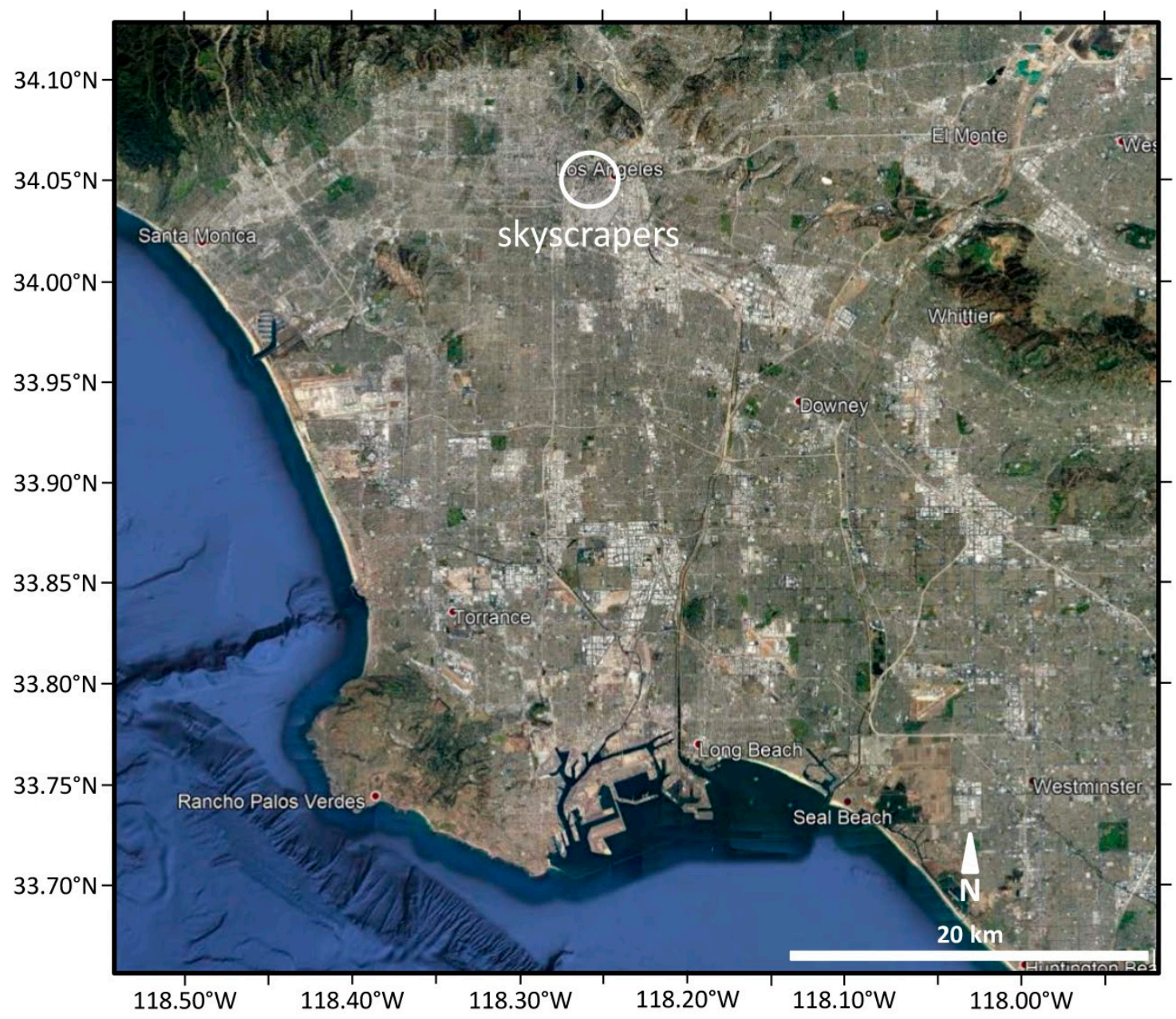

Figure 9. Map of Los Angeles (background image: Google).

\subsection{Greater Cairo}

The Greater Cairo area (Figure 10) is the largest metropolitan area in Egypt and the whole Africa. It consists of cities such as Cairo, Giza, Helwan, and Shubra El Kheima with their surroundings. Greater Cairo covers approximately an area of $1790 \mathrm{~km}^{2}$ and includes a variety of land uses associated with a complex mix of land cover, such as a central business district, urban/suburban residential areas, and some rural areas [26]. Similar to other ancient Egyptian cities, often linked in the past to military camps, we can notice areas presenting gridded patterns in their spatial structure within the boundaries of the city [20]. Cairo encountered rapid urban growth, which can be noticed not only in the expansion of legal housing, mainly in the area of Cairo and Giza, but also from informally urbanized areas. These areas are dominated by the illegal slums mainly in the plateaus around old Cairo city and over the cultivated lands all over the Greater Cairo Area [26]. 


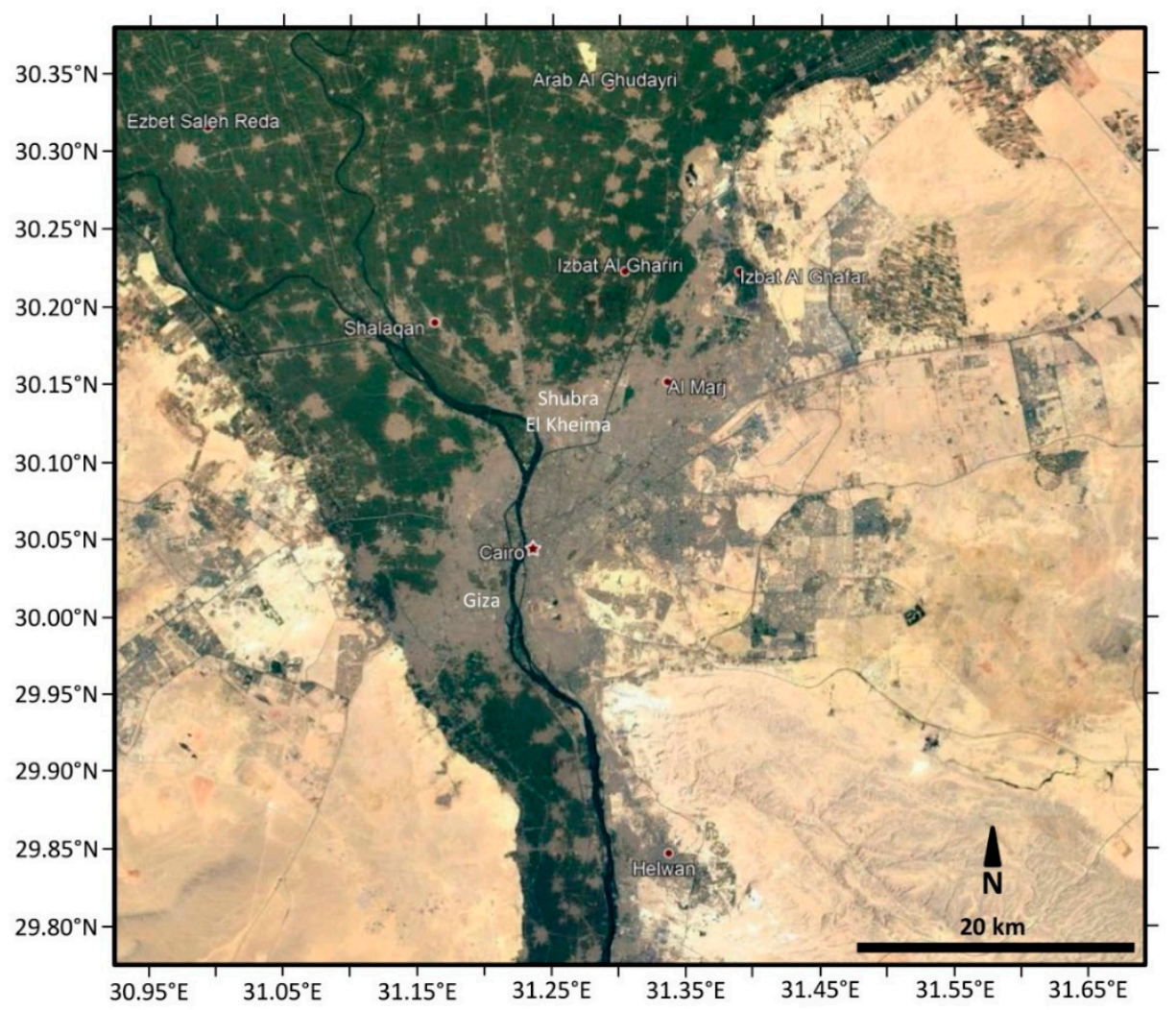

Figure 10. Map of the Greater Cairo area (background image: Google).

\section{Dataset and Methods}

In this study, we used ALOS PALSAR-1 data as it is a freely and easily accessible full- polarimetric dataset that could be found over selected study areas. ALOS PALSAR provides data with a similar resolution to the WSF Evolution provided by DLR, which was used, as stated before, as a reference layer for the extraction of urban areas. The WSF Evolution was processed by the German Aerospace Center (DLR) using Google Earth Engine (GEE) [27,28]. More detailed information on the datasets used in this study is available in Table 1.

Table 1. Detailed information about the sensor, acquisition day, satellite data center, and frame or path/row information of the dataset employed.

\begin{tabular}{|c|c|c|c|c|}
\hline City & Data & Acquisition Day & Data Provider & Path/Frame \\
\hline \multirow{2}{*}{ Beijing } & ALOS PALSAR & 02/04/2009 & ASF & \multirow{2}{*}{$443 / 790$} \\
\hline & WSF layer & 2009 & DLR & \\
\hline \multirow{2}{*}{ Shanghai } & ALOS PALSAR & 29/03/2011 & ASF & \multirow{2}{*}{$437 / 620$} \\
\hline & WSF layer & 2011 & DLR & \\
\hline \multirow{2}{*}{ Milan } & ALOS PALSAR & $24 / 03 / 2009$ & ESA & \multirow{2}{*}{$640 / 900$} \\
\hline & WSF layer & 2009 & ASF & \\
\hline \multirow{2}{*}{ New York } & ALOS PALSAR & $01 / 04 / 2011$ & ASF & \multirow{2}{*}{$126 / 810$} \\
\hline & WSF layer & 2011 & DLR & \\
\hline \multirow{2}{*}{ San Francisco } & ALOS PALSAR & $11 / 11 / 2009$ & ASF & \multirow{2}{*}{$218 / 750$} \\
\hline & WSF layer & 2009 & DLR & \\
\hline \multirow{2}{*}{ Los Angeles } & ALOS PALSAR & $16 / 03 / 2009$ & ASF & \multirow{2}{*}{$212 / 670$} \\
\hline & WSF layer & 2009 & DLR & \\
\hline \multirow{2}{*}{ Greater Cairo } & ALOS PALSAR & $16 / 11 / 2006$ & ESA & \multirow{2}{*}{$603 / 600$} \\
\hline & WSF layer & 2006 & DLR & \\
\hline
\end{tabular}


We used the four-component Yamaguchi decomposition (described in Section 1) of the fully-polarimetric dataset to analyze different backscatter mechanisms over urban areas. For the extraction of urban extent, we used a mask layer from World Settlement Footprint Evolution (Figure 11).

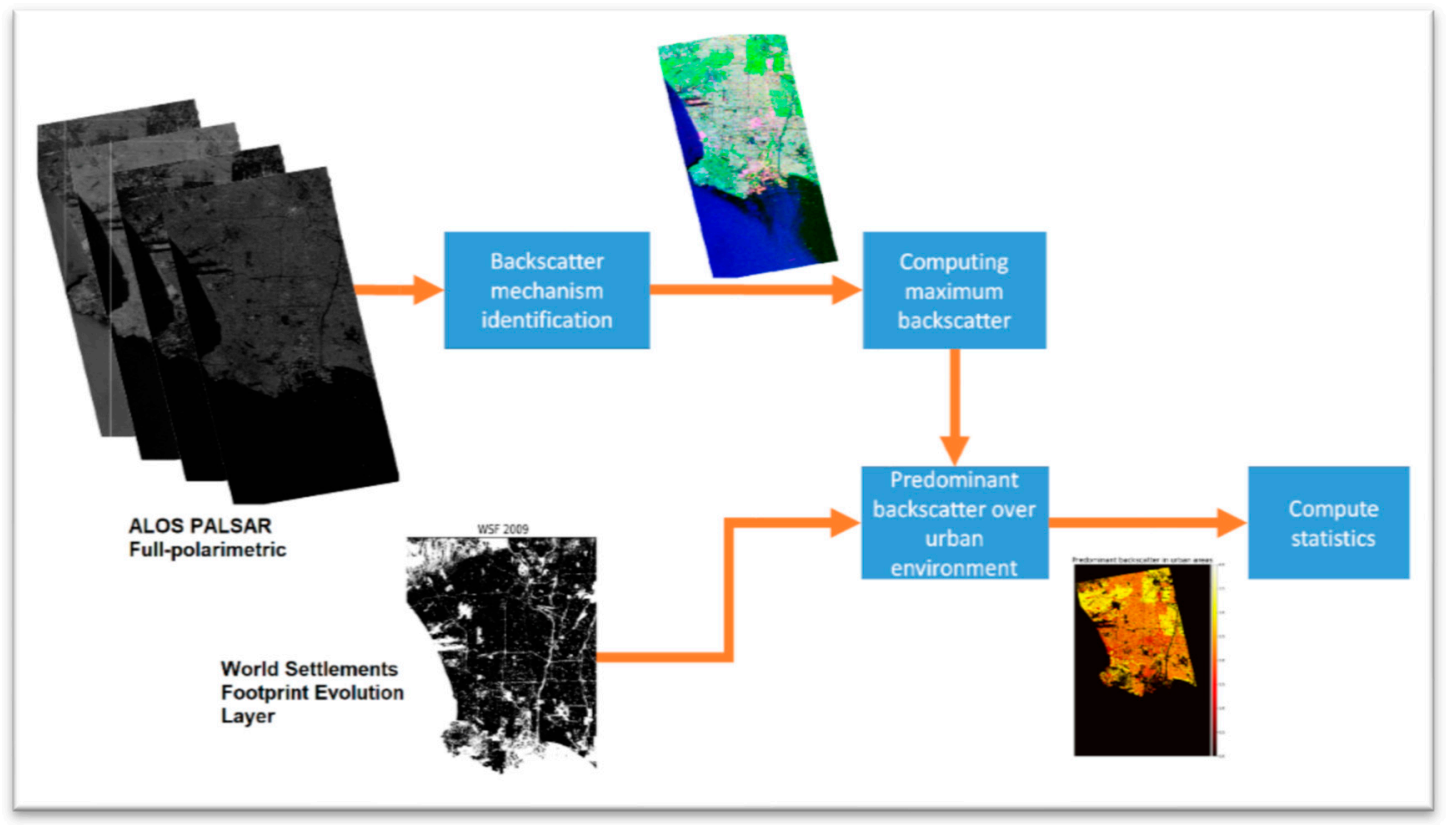

Figure 11. Workflow for the predominant backscatter identification over urban areas.

The first step was to identify different backscatter mechanisms in ALOS PALSAR scenes. We processed the data following the processing workflow using PolSARpro software [9]: (i) data import; (ii) data extraction; (iii) coherency matrix (T3) generation; (iv) Yamaguchi four-component decomposition; and (v) geocoding, as indicated in Figure 12.

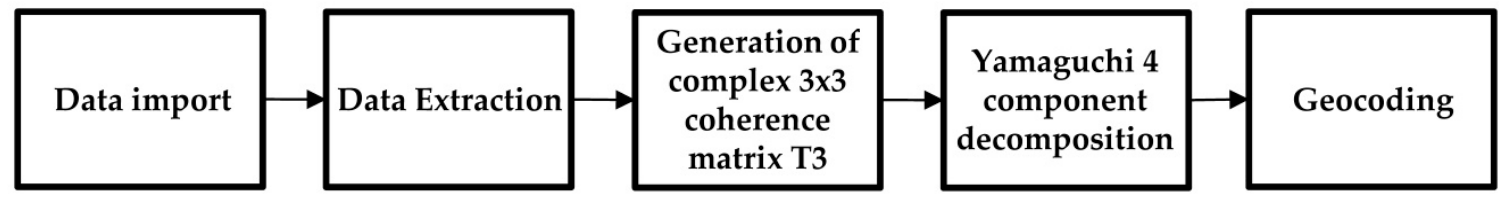

Figure 12. Processing workflow applied to the Synthetic Aperture Radar (SAR) polarimetric data.

In the following step, we computed the maximum backscatter value for each pixel from the retrieved four-backscatter components, identifying the predominant backscatter pixel-wise. The predominant backscatter was then masked with the urban footprint layer obtained from WSF Evolution. The WSF Evolution contains urban extent classified for each year starting from 1985 until 2015; therefore, for masking, we chose the layers matching the acquisition dates.

Finally, after computing the predominant backscatter mechanism for urban areas, we computed the percentage of urban areas having specific backscatter mechanisms, i.e., single, double, volume/random, and helix scatter mechanisms obtained with the Yamaguchi four-component decomposition.

\section{Results}

Figure 13A, Figure 14A, Figure 15A, Figures 16A, 17A, 18A and 19A show the RGB false composition created using three of the four-backscatter mechanisms obtained in the Yamaguchi polarimetric decomposition (the full results of the Yamaguchi four-component decomposition for all the analyzed cities can be found in Annex in Figures A1 and A2). The assignment of colors is as follows: red corresponds to double bounce, green to volume scattering, and blue to surface scattering-single 
bounce. Subsequently, Figure 13B, Figure 14B, Figure 15B, Figure 16B, Figure 17B, Figure 18B and Figure 19B depict the WSF layer for each city, and the computed predominant backscatter over urban areas is presented in Figure 13C, Figure 14C, Figure 15C, Figure 16C, Figure 17C, Figure 18C and Figure 19C.
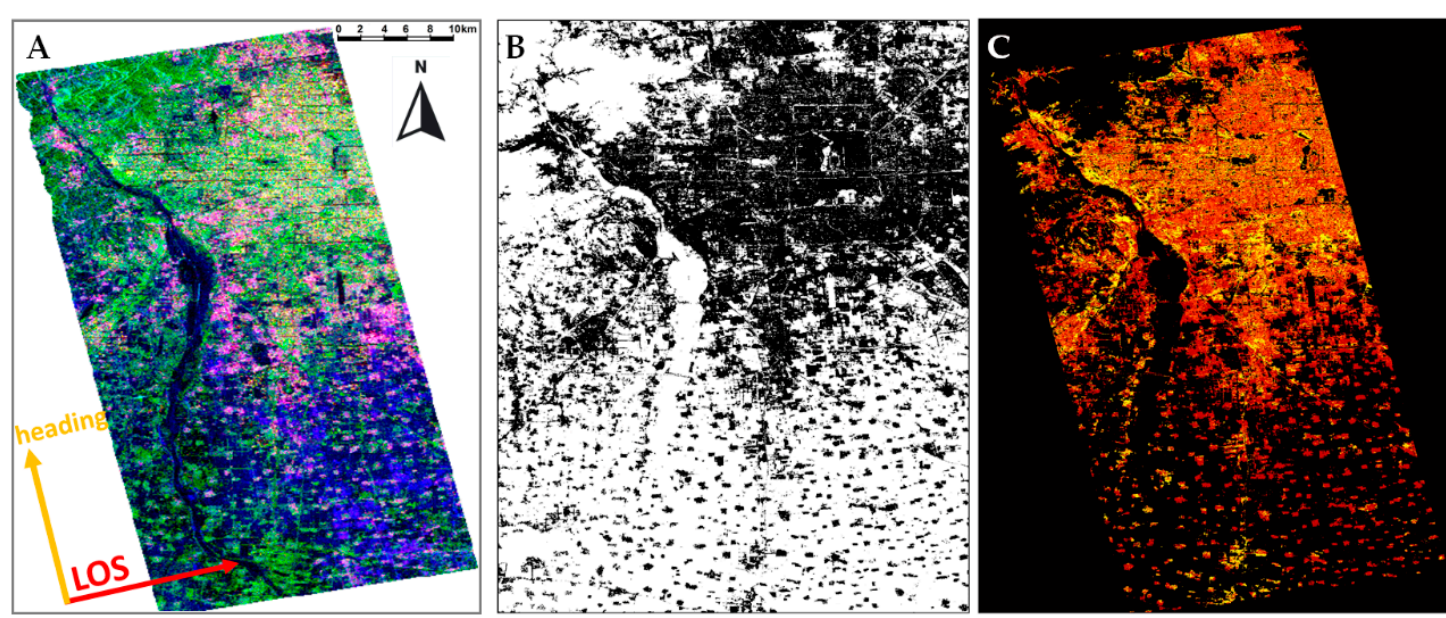

Helix

Volume

Double

Single

Figure 13. Yamaguchi decomposition false RGB (A), World Settlement Footprint (WSF) derived for 2006 (B), and predominant backscatter in the urban area of Beijing (C). The red arrow indicates the Line-of-Sight (LOS) and the orange arrow the satellite heading.
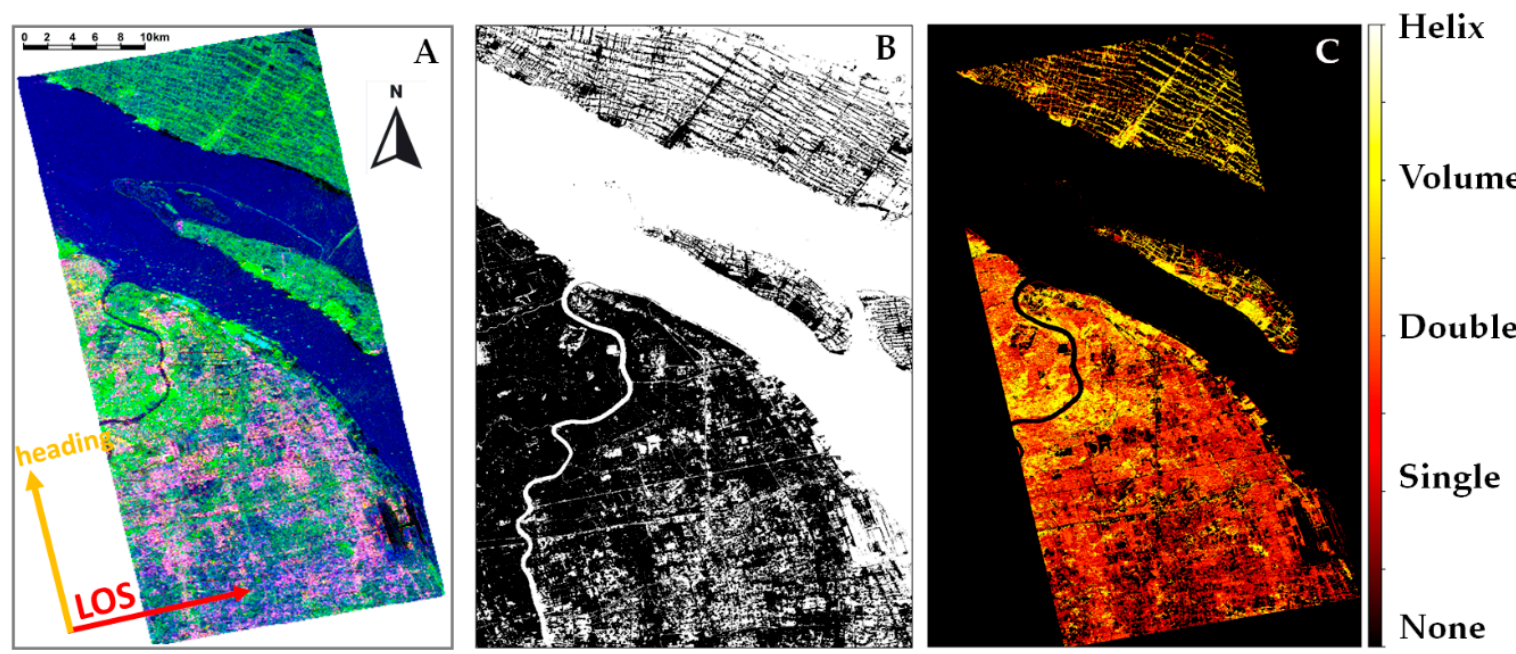

Figure 14. Yamaguchi decomposition false RGB (A), WSF derived for 2011 (B), and predominant backscatter in urban area of Shanghai (C). The red arrow indicates the Line-of-Sight (LOS) and the orange arrow the satellite heading. 



Helix

Volume

Double

Figure 15. Yamaguchi decomposition false RGB (A), WSF derived for 2009 (B), and predominant backscatter in urban area of Milan (C). The red arrow indicates the Line-of-Sight (LOS) and the orange arrow the satellite heading.
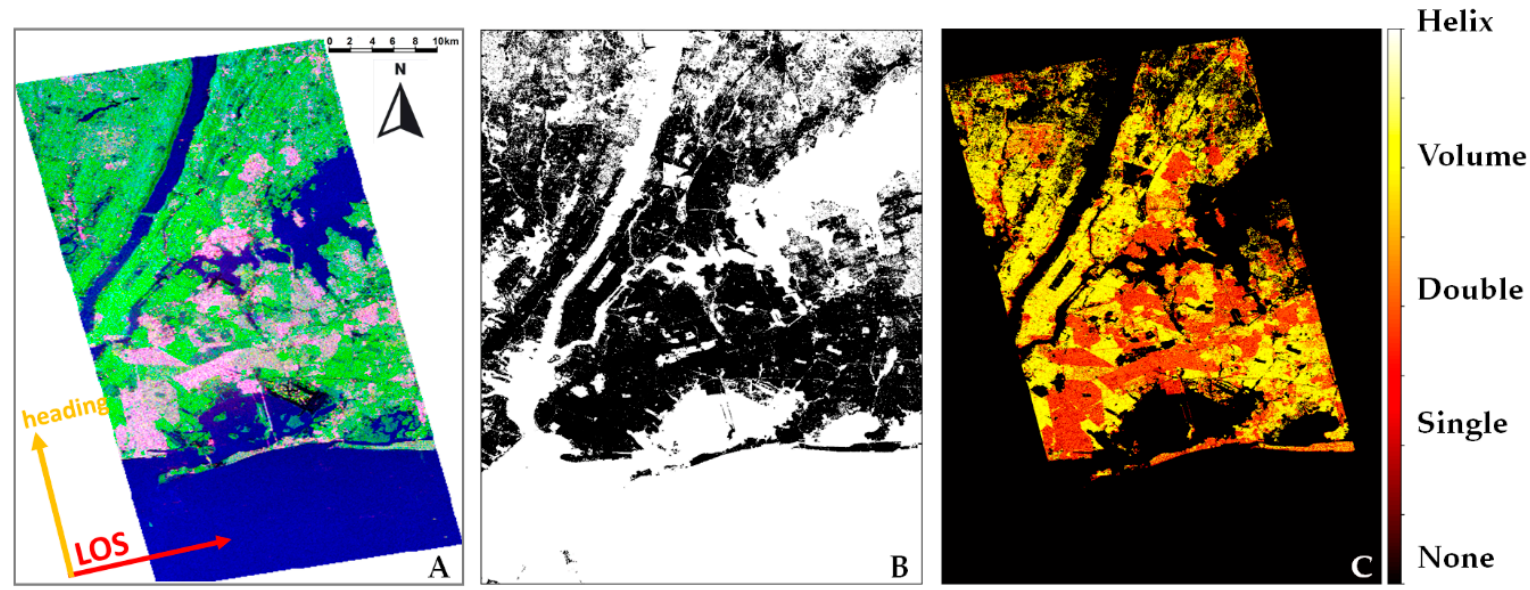

Figure 16. Yamaguchi decomposition false RGB (A), WSF derived for 2011 (B), and predominant backscatter in the urban area of New York (C). The red arrow indicates the Line-of-Sight (LOS) and orange arrow the satellite heading.
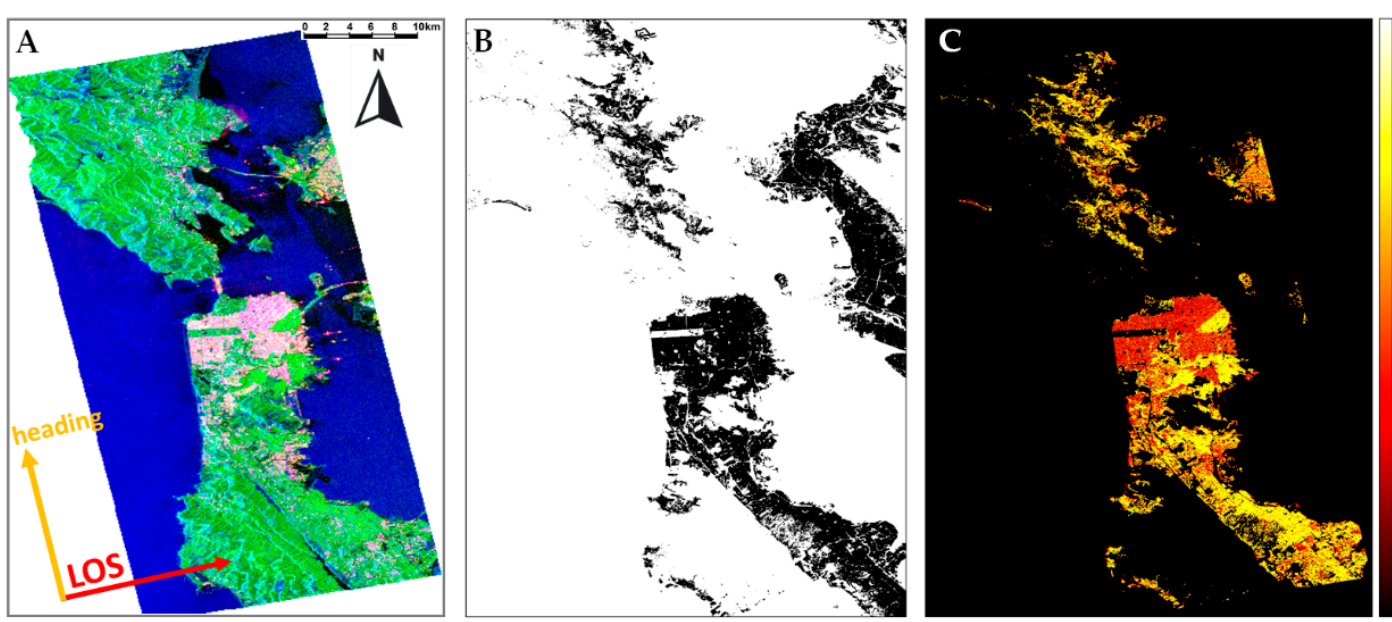

Helix

Volume

Double

Single

None

Figure 17. Yamaguchi decomposition false RGB (A), WSF derived for 2009 (B), and predominant backscatter in urban area of San Francisco (C). The red arrow indicates the Line-of-Sight (LOS) and the orange arrow the satellite heading. 

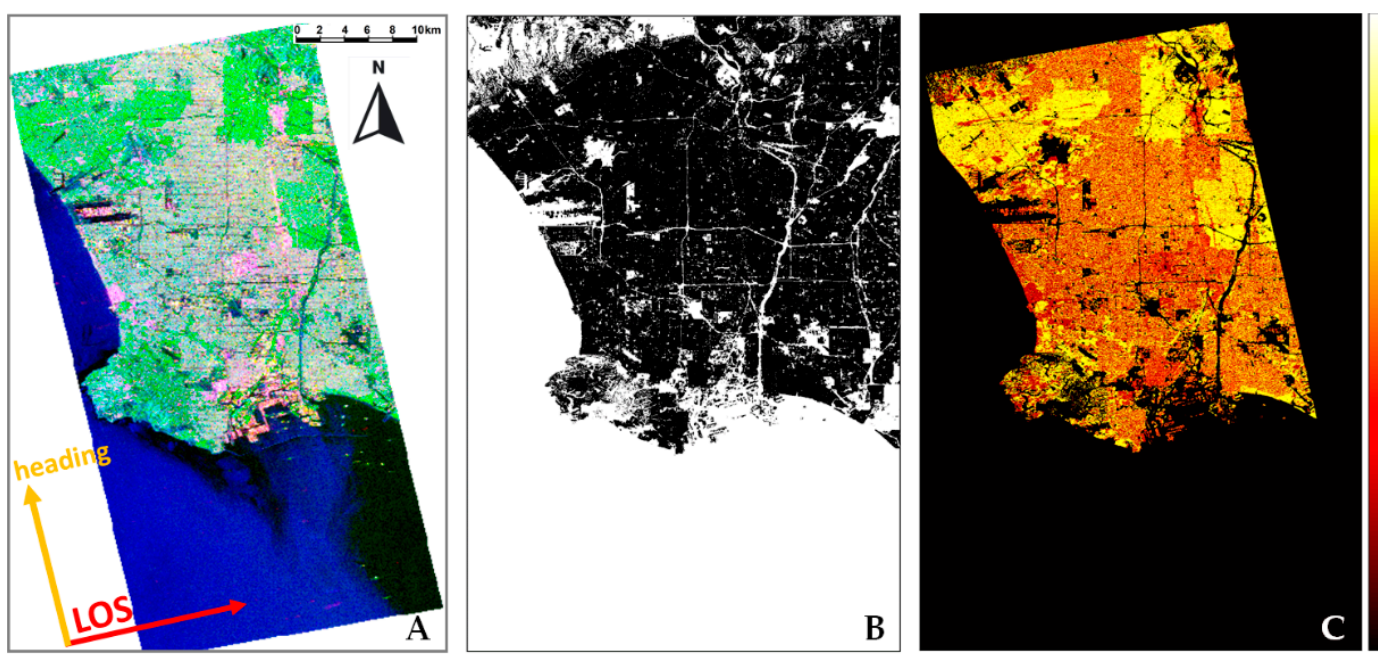

Helix

Volume

Double

Figure 18. Yamaguchi decomposition false RGB (A), WSF derived for 2009 (B), and predominant backscatter in urban area of Los Angeles (C). The rted arrow indicates the Line-of-Sight (LOS) and the orange arrow the satellite heading.
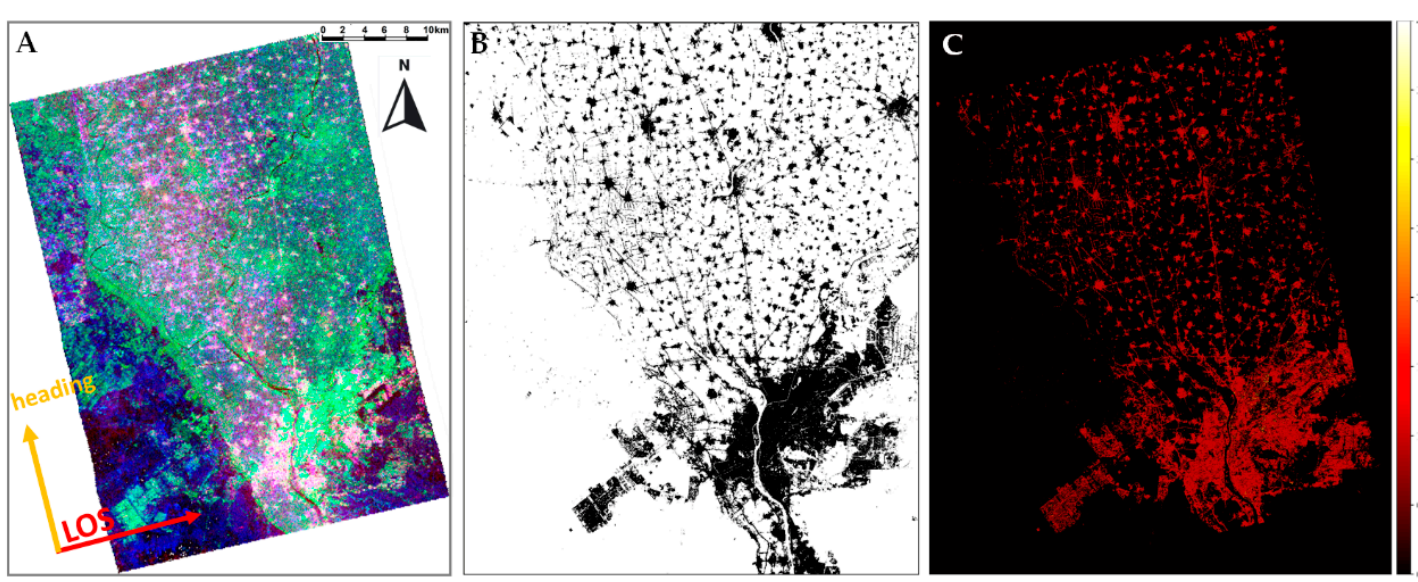

Helix

Volume

Double

Single

Figure 19. Yamaguchi decomposition false RGB (A), WSF derived for 2006 (B), and predominant backscatter in urban area of Greater Cairo (C). The red arrow indicates the Line-of-Sight (LOS) and the orange arrow the satellite heading.

From the false RGB composite of Beijing Figure 13A we can notice that in the central area of the city the predominant backscatter is double bounce mixed with volume scattering-seen in yellow and green in 13A. This is related to the characteristics of the inner city where streets are well organized, with wide main streets, and buildings often oriented in an orthogonal fashion to the satellite looking direction. We can also distinguish zones were the predominant backscatter is double and single bounce (Figure 14A, in magenta), which is the case in the presence of taller and more sparse buildings.

Similar features and backscatter characteristics can be observed in Shanghai, especially where the streets are rotated from the cardinal directions. The mix of volume and double bounce scattering (Figure 14A, in green) can be observed in, for example, Pudong district on the eastern bank of the Huangpu River. In many parts of the city, the volume scattering is overestimated, mainly due to the building rotation or presence of short, densely located buildings. A further discussion of this example is presented in Section 4.

The mix of double bounce and volume scattering in the strict center is also present in the case of the city of Milan (Figure 15). Volume scattering is evident along the main routes extending in straight lines from the center, diverging like rays. The characteristic, circular shape of the main streets 
(along city rings) is shaping the direction of building locations. For buildings located on the streets slightly rotated from the cardinal directions, we can observe a domination of single and double bounce, whereas, for those greatly rotated, the volume backscatter dominates.

Moreover, in the case of New York, the orientation of streets determines the type of predominant observed backscatter (Figure 16). A good example here is Manhattan, where the predominant backscatter is volume scattering; only for the tallest buildings located next to the shore can we also observe double bounce. Double bounce is also present in some part of Brooklyn and Queens, where the position of building facades mostly follows the cardinal directions.

This phenomenon is visible also in the case of the previously mentioned San Francisco (Figure 17). Here, the predominant backscatter in all the residential areas, especially in the eastern part, is double bounce due to the wide roads, the regular grid pattern of streets, and the main streets' orientation in cardinal directions. However, the western part of the city has more influences of other backscattering mechanisms because of its architecture-smaller buildings mixed with vegetation and narrower streets.

The financial district in the NE part of the city can be characterized by high values of double bounce, whereas the South of Market zone displays predominant volume scattering caused by the rotation of buildings $\left(0^{\circ}<\alpha<45^{\circ}\right)$ relative to the track of the satellite. On the eastern side of the bay, we can identify large elongated area exhibiting volume scattering - it is the Golden Gate Park consisting mainly of trees and grass.

As mentioned in Section 1, Los Angeles is another agglomeration located in North America that follows the land ordinance according to which the land was divided into regular squares and the main streets follow the cardinal directions. Due to this fact, in the major part of the city, apart from some of the zones downtown, the predominant backscatter mechanism is double bounce (Figure 18). Double bounce can also be observed in the Port of Long Beach. Here, the large area of the containers terminal is dominated by double bounce due to large streets between the rows of containers and the sparser location of containers.

In all the examples presented above, we can distinguish various scattering mechanisms present in different parts of urban agglomerations. This is not the case for Greater Cairo, where the vast majority of the urban area is dominated by a single bounce scattering mechanism (Figure 19). This effect is due to the specific urban structure of Cairo, with narrow streets and very dense buildings, many of which have flat roofs. Although the predominant backscatter is single bounce, indicating surface scattering from buildings, the contribution of other scattering mechanisms is not negligible but in the same order of magnitude.

The street orientation also has an impact, resulting in an overestimation of volume scattering, appearing green in Figure 19A.

In addition to the qualitative analysis, we have quantified the percentage of each backscatter mechanism for the different cities and summarized it in Table 2. It can be clearly noticed that, although for urban areas the theoretical assumption is that the principal backscatter mechanism is double bounce, the analyzed cities do not hold this assumption. In most cases, we are dealing with a mix of all the three main mechanisms (single, double, and volume scattering) with an overestimation of the latter due to the buildings rotation with respect to the line of sight of the satellite. 
Table 2. Percentage of the different backscatter mechanisms measured over urban pixels using ALOS PALSAR full-polarization data. Urban pixels extracted from the WSF layer for the same year of the ALOS PALSAR acquisition.

\begin{tabular}{ccccc}
\hline City & $\begin{array}{c}\text { Single Bounce } \\
{[\%]}\end{array}$ & $\begin{array}{c}\text { Double Bounce } \\
{[\%]}\end{array}$ & $\begin{array}{c}\text { Volume/Random } \\
\text { Scattering [\%] }\end{array}$ & $\begin{array}{c}\text { Helix Scattering } \\
{[\%]}\end{array}$ \\
\hline Beijing & 54.42 & 21.78 & 23.77 & 0.03 \\
Shanghai & 52.29 & 18.11 & 29.58 & 0.02 \\
Milan & 48.31 & 21.24 & 30.42 & 0.03 \\
New York & 22.62 & 20.11 & 57.24 & 0.03 \\
San Francisco & 31.35 & 10.27 & 58.37 & 0.01 \\
Los Angeles & 34.73 & 12.42 & 52.83 & 0.02 \\
Greater Cairo & 98.86 & 0.15 & 0.99 & 0.00 \\
\hline
\end{tabular}

\section{Discussion}

As mentioned in Section 1, the type of dominant backscatter in urbanized environments depends on many factors, e.g., the building rotation angle with respect to the radar looking direction. In the examples shown in Section 2, we can observe that build-up areas following or being slightly rotated from cardinal directions tend to exhibit double bounce mixed with single bounce. With larger rotation angles, we can observe the overestimation of volume scattering. This phenomenon is depicted in Figure 20 presenting details over New York. The zone highlighted with a violet ellipse is the zone with dominating double and single bounce scattering, while the areas with a yellow ellipse are dominated by volume scattering.
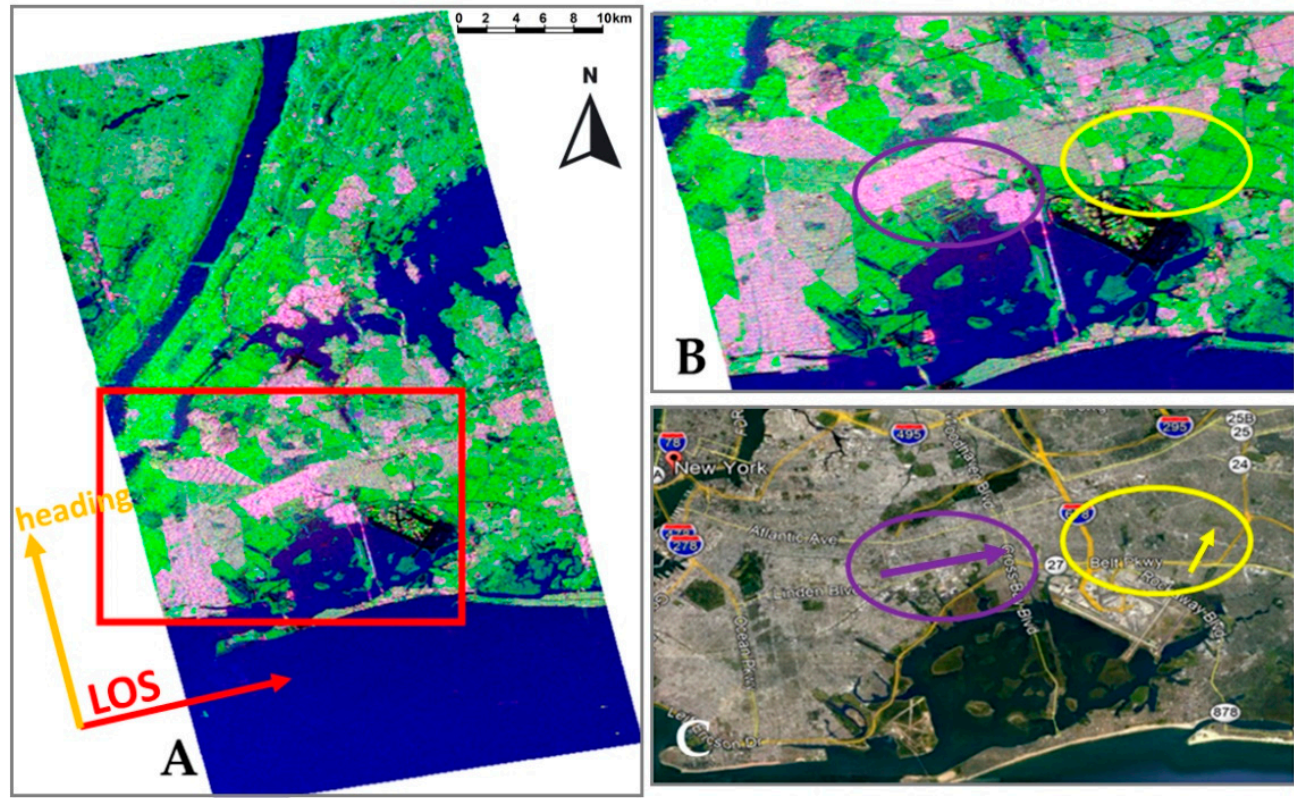

Figure 20. Details of Yamaguchi decomposition false RGB over New York (A), the zoom of area highlighted within the red rectangle (B), and the Google Earth image over the zoomed area (C).

The same effect can be noted in the well-known San Francisco case, as mentioned previously. In Figure 21, we can distinguish two characteristic zones for San Francisco area-the financial district (violet ellipse) where the main streets are following more or less cardinal directions, and the south of market zone (yellow ellipse) with rotated buildings and the predominant volume scattering. The arrows in Figure 21C follow the street orientations in the aforementioned districts. 

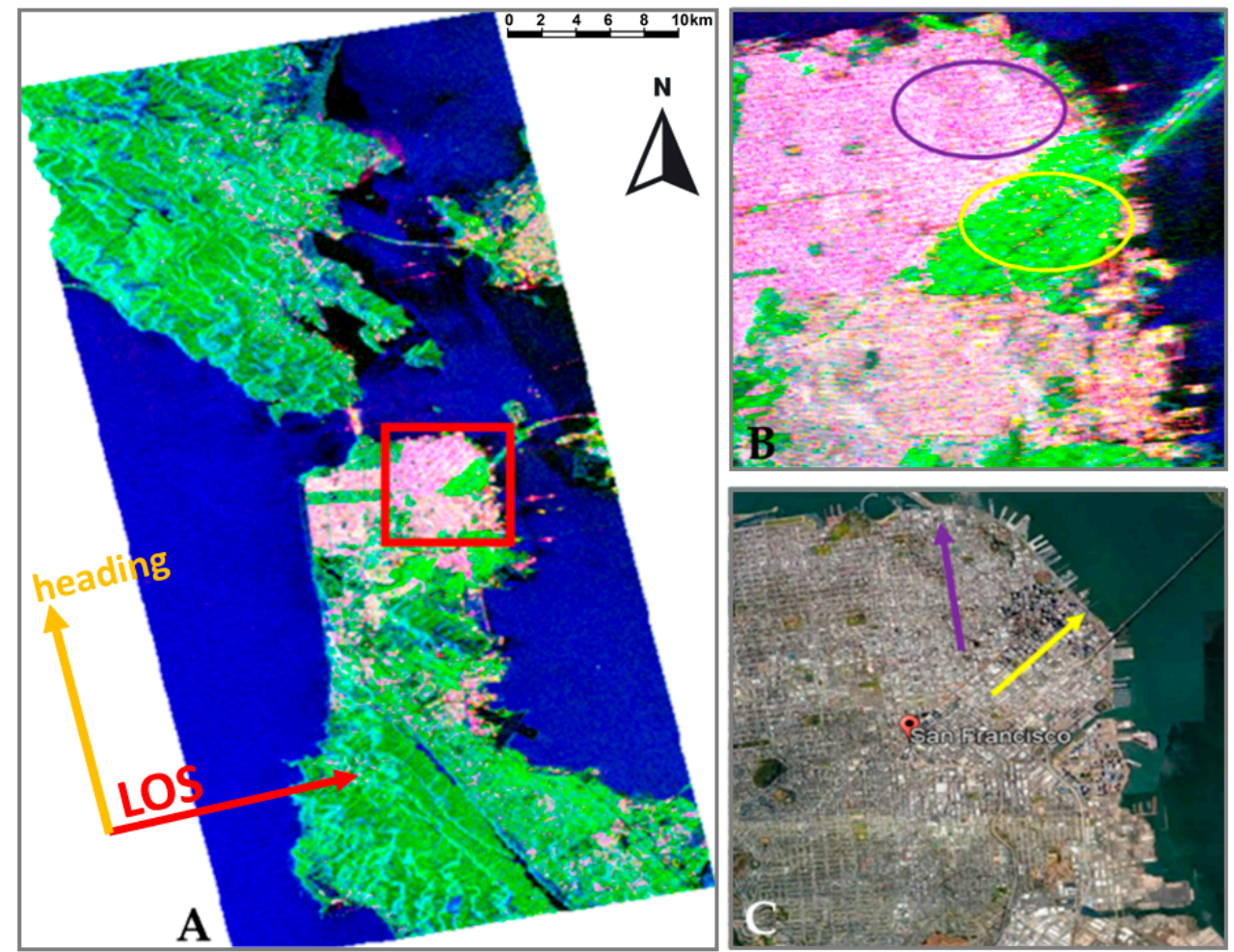

Figure 21. Details of Yamaguchi decomposition false RGB over San Francisco (A), the zoom of area highlighted within the red rectangle (B), and the Google Earth image over the zoomed area (C).

Other factors influencing the type of identified predominant backscatter are the buildings heights and density of build-up areas. These can be observed in the example of Beijing (Figure 22), where the radar response from high and sparse buildings is dominated by double bounce (yellow ellipse).
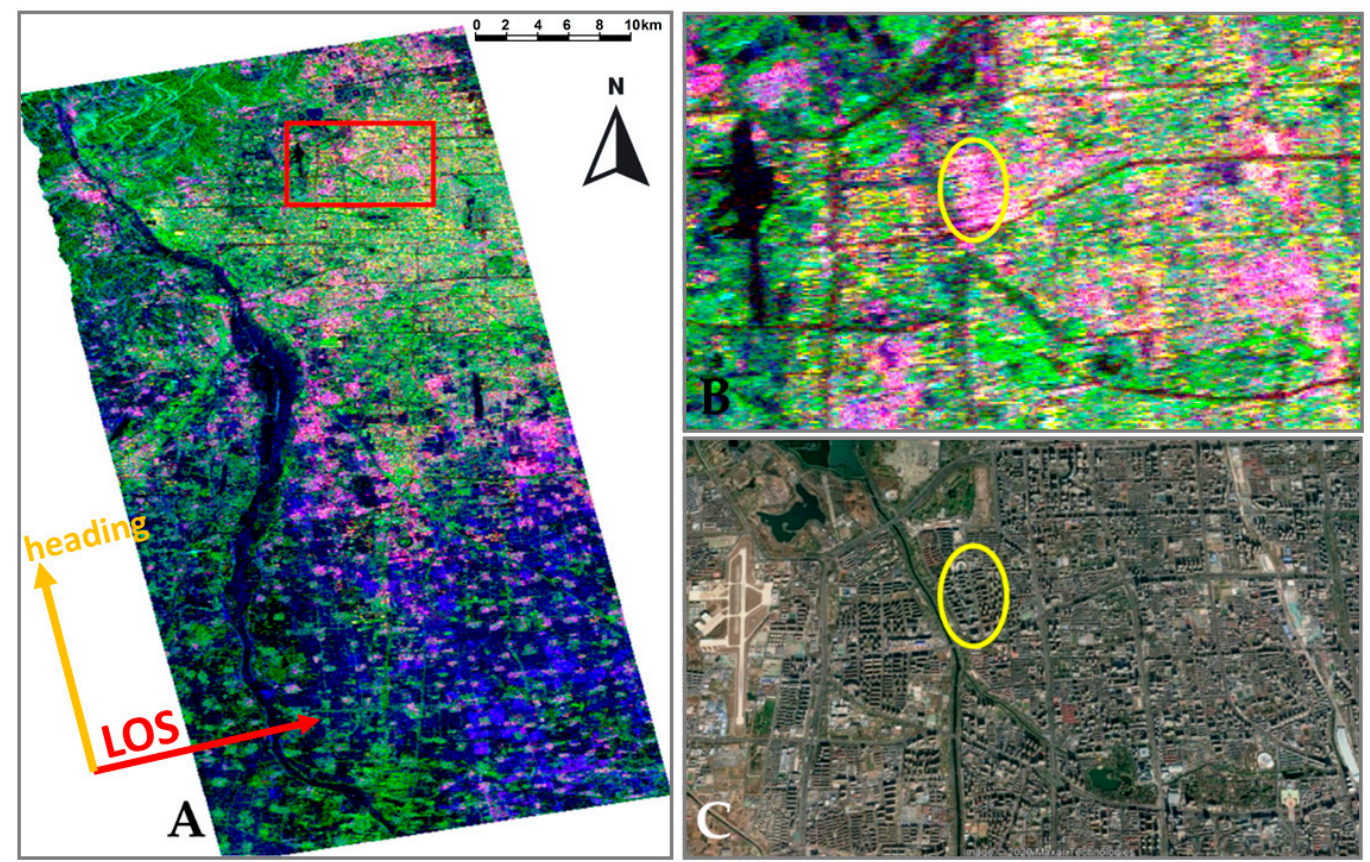

Figure 22. Details of Yamaguchi decomposition false RGB over Beijing (A), the zoom of area highlighted within the red rectangle $(\mathbf{B})$, and the Google Earth image over the zoomed area $(\mathbf{C})$. 
On the other hand, in the case of Shanghai, in the northern part of Pudong district, in the port, we can identify areas with the predominant single bounce scattering (Figure 23, highlighted with red ellipses). This relates to the presence of big containers in the harbor surrounded by concrete pavement, providing increased surface scattering.
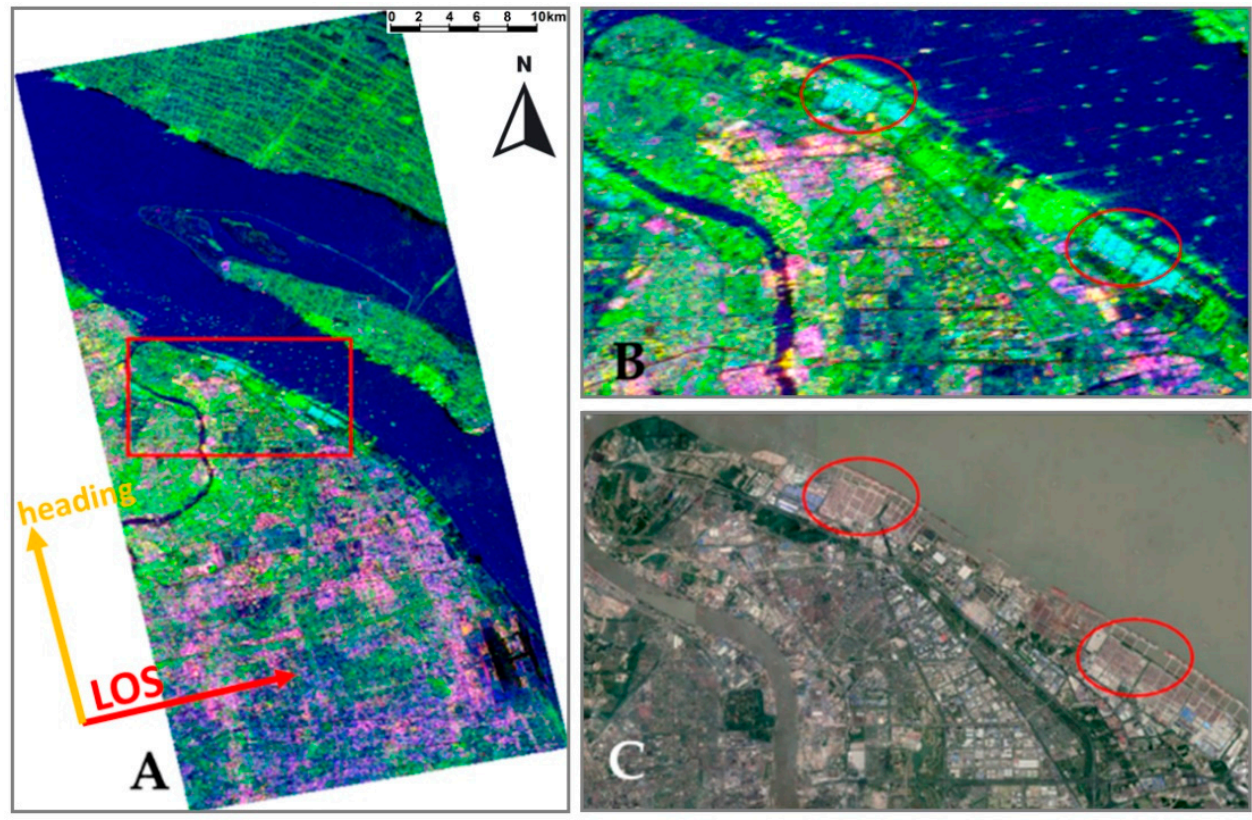

Figure 23. Details of Yamaguchi decomposition false RGB over Shanghai (A), the zoom of area highlighted within the red rectangle (B), and the Google Earth image over the zoomed area (C).

In addition, another factor that could influence a reliable recognition of scattering mechanisms is the spatial resolution of the data used in the analysis. In order to briefly illustrate the impact of resolution on the identification of scattering mechanisms, we processed ALOS PALSAR-2 and RADARSAT-2 full-polarimetric data over San Francisco to evaluate if the utilization of higher resolution data could help to identify more double bounce response over built-up areas than with the medium resolution data of ALOS PALSAR. Details of the acquisitions used in this analysis are presented in Table 3.

Table 3. Characteristics of the full-polarimetric datasets employed over San Francisco.

\begin{tabular}{ccccc}
\hline Satellite & Acquisition Date & $\begin{array}{c}\text { Frequency Band } \\
\text { (GHz) }\end{array}$ & $\begin{array}{c}\text { Spatial Resolution } \\
\text { [m] }\end{array}$ & $\begin{array}{c}\text { Incident Angle } \\
\text { [degrees] }\end{array}$ \\
\hline ALOS PALSAR 1 & $11 / 11 / 2009$ & $\mathrm{~L}(1.270)$ & $40 \times 40$ & 23.87 \\
ALOS PALSAR 2 & $24 / 03 / 2015$ & $\mathrm{~L}(1.236)$ & $6 \times 6$ & 33.87 \\
RADARSAT-2 & $09 / 04 / 2008$ & $\mathrm{C}(5.404)$ & $8 \times 8$ & 28.9 \\
\hline
\end{tabular}

Looking at Figure 24, we can conclude that the area of rotated skyscrapers appears in green for all Yamaguchi RGBs, meaning that a higher spatial resolution does not influence the recognition of double bounce mechanism over this area. Nevertheless, the intensity of magenta increased for ALOS PALSAR 2 case, which may be interpreted as an increase in the double bounce mechanism (higher values in red channel). Obviously, higher resolution data allow one to better isolate and identify ground targets and scattering mechanisms associated with them. Working with high resolution or very high resolution SAR data can introduce, however, multipath scattering visible for man-made objects such as high buildings and bridges [29] 

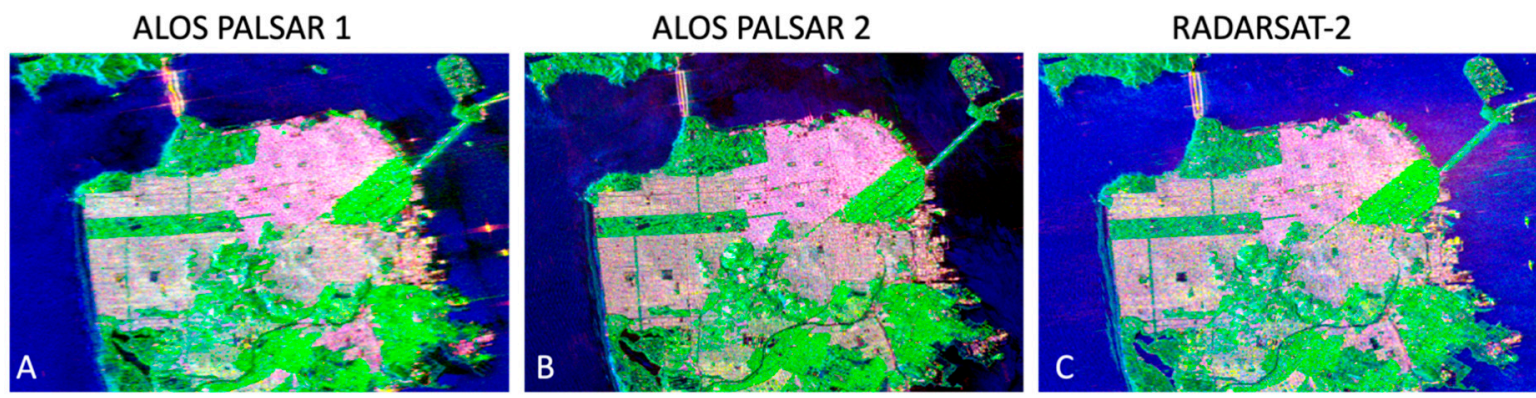

Figure 24. Yamaguchi RGB false color of San Francisco area with ALOS PALSAR 1 (A), ALOS PALSAR 2, (B) and RADARSAT-2 (C).

Finally, we have summarized the percentage of the predominant backscatter mechanism in the urban area for the different datasets over San Francisco (Table 4). Looking at the values obtained, higher resolution data help to increase the double bounce mechanism from almost $15 \%$ up to almost $31 \%$ in the case of ALOS PALSAR 2 and up to $24 \%$ for RADARSAT-2. However, the volume/random mechanism predominates all over the scene, being the predominant backscatter mechanism for all cases, in more than $40 \%$ of the analyzed area. The increase of double bounce values is linked with the reduction in single bounce, and only a small part of the volume scattering. This last phenomenon could also be related to the higher incidence angle of the ALOS PALSAR 2 data (over 33 degrees) with respect to the ALOS PALSAR 1 (over 23 degrees).

Table 4. Percentage of the different backscatter mechanisms measured over urban pixels using different full-polarization data. The urban pixels were extracted from the WSF layer for the same year of each correspondent acquisition.

\begin{tabular}{ccccc}
\hline Scattering Mechanism Sensor & Single Bounce [\%] & Double Bounce [\%] & Volume/Random [\%] & Helix [\%] \\
\hline ALOS PALSAR-1 & 36.68 & 14.87 & 48.43 & 0.02 \\
RADARSAR-2 & 34.53 & 24.15 & 40.8 & 0.52 \\
ALOS PALSAR-2 & 23.31 & 30.92 & 45.68 & 0.09 \\
\hline
\end{tabular}

\section{Conclusions}

As is widely mentioned in the literature, theoretically, the principal backscatter mechanism from buildings and man-made objects is double bounce. This rule is true when we are dealing with isolated objects or we are analyzing backscatter using high-resolution SAR polarimetric data. In practice, when using medium-resolution data there are many factors that may influence the backscatter and make it more complex. These factors are, among others, the orientation of the buildings with respect to satellite flight direction, the dimensions of the streets, the type of construction (i.e., numerous planes of the roof), etc. In this work, we have analyzed the influence of these factors on the backscatter mechanism for various types of urban agglomerations, in various locations, and for different types of urban structures-irregular and regular-with narrow and wide streets, etc. It has to be noted that, in all cases, we can identify the strong effect of building rotation with respect to satellite flight path on the predominant backscatter mechanism.

The outcome of this study is significant to the scientific community working with medium resolution polarimetric data as it brings a new understanding to the applicability of polarimetric principles using medium-resolution data in different urban environments, such as the analyzed cities. We can conclude that the theoretical assumption of double bounce predominance for buildings does not apply to cities with irregular layout and narrow streets, especially in medium-resolution polarimetric studies. Cities with wider streets following a regular grid, such as Beijing, Milan, or New York, provide the highest percentage of double bounce predominance among the analyzed cities. 
Comparing medium- and high-resolution data with the same wavelength, we can conclude that high-resolution data help in increasing the amount of pixels with double bounce predominance, as single pixels may be filled with individual buildings, converging to what theory establishes.

All the factors mentioned above should be taken into consideration when performing urban area detection based only on polarimetric data and standard algorithms.

Author Contributions: Conceptualization, J.M.D.B.; M.F. and J.P.; Formal analysis, J.M.D.B.; M.F.; and J.P.; Investigation, J.M.D.B. and M.F.; Methodology, J.M.D.B.; M.F. and J.P.; Resources, A.M.R.-A. and M.M.; Software, J.M.D.B.; Validation, J.M.D.B. and M.F.; Visualization, J.M.D.B. and M.F.; Writing - original draft, J.M.D.B. and M.F.; Writing - review \& editing, J.M.D.B; M.F.; J.P.; A.M.R.-A. and M.M. All authors have read and agree to the published version of the manuscript.

Funding: This research received no funding.

Acknowledgments: The authors want to acknowledge the use of the satellite data employed in this work, i.e. ALOS PALSAR (Copyright JAXA, METI [2006-2011] and ESA/JAXA [2006-2011]. Research was supported by PAIUJA-2019/2020 and CEACTEMA from University of Jaén (Spain), and RNM-282 research group from the Junta de Andalucía (Spain).

Conflicts of Interest: The authors declare no conflict of interest.

\section{Appendix A}

In this appendix, the reader can find each of the Yamaguchi four-component decomposition for each of the analyzed cities presented in this study. It is worth noting that the values of the Helix scattering are always lower than the others, reaching sometimes even $10 \mathrm{~dB}$ less than the single, double, and volume scattering. Figures A1 and A2 present results for cities in alphabetic order. Different background colour is used to highlight various types of scattering - single bounce (cyan), double bounce (yellow), volume (green) and helix (violet).
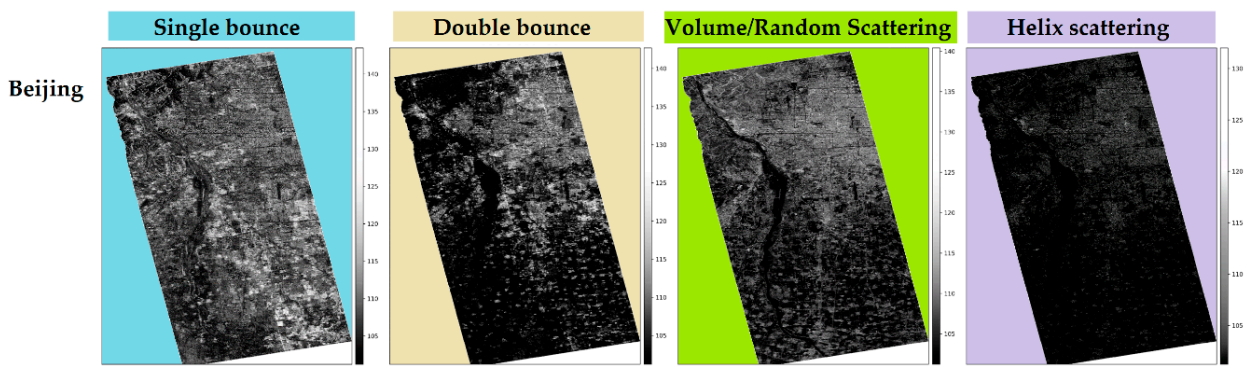

Cairo
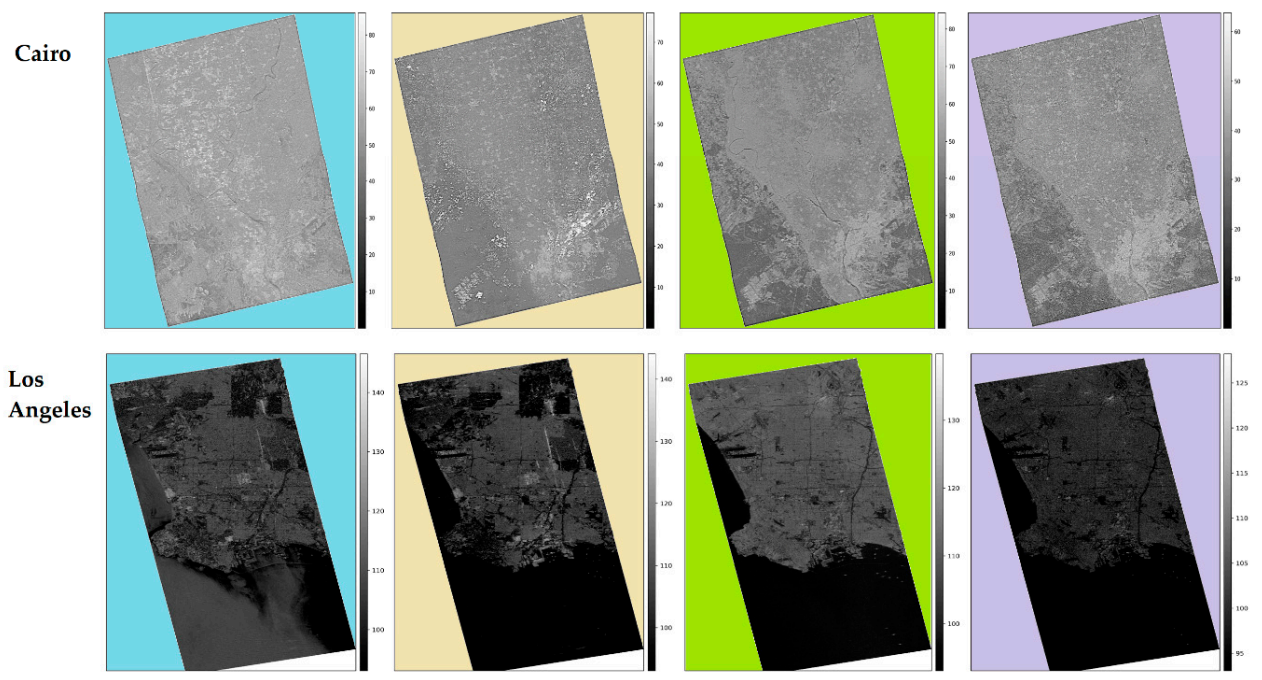

Figure A1. Yamaguchi four-component for Beijing, Greater Cairo, and Los Angeles. 


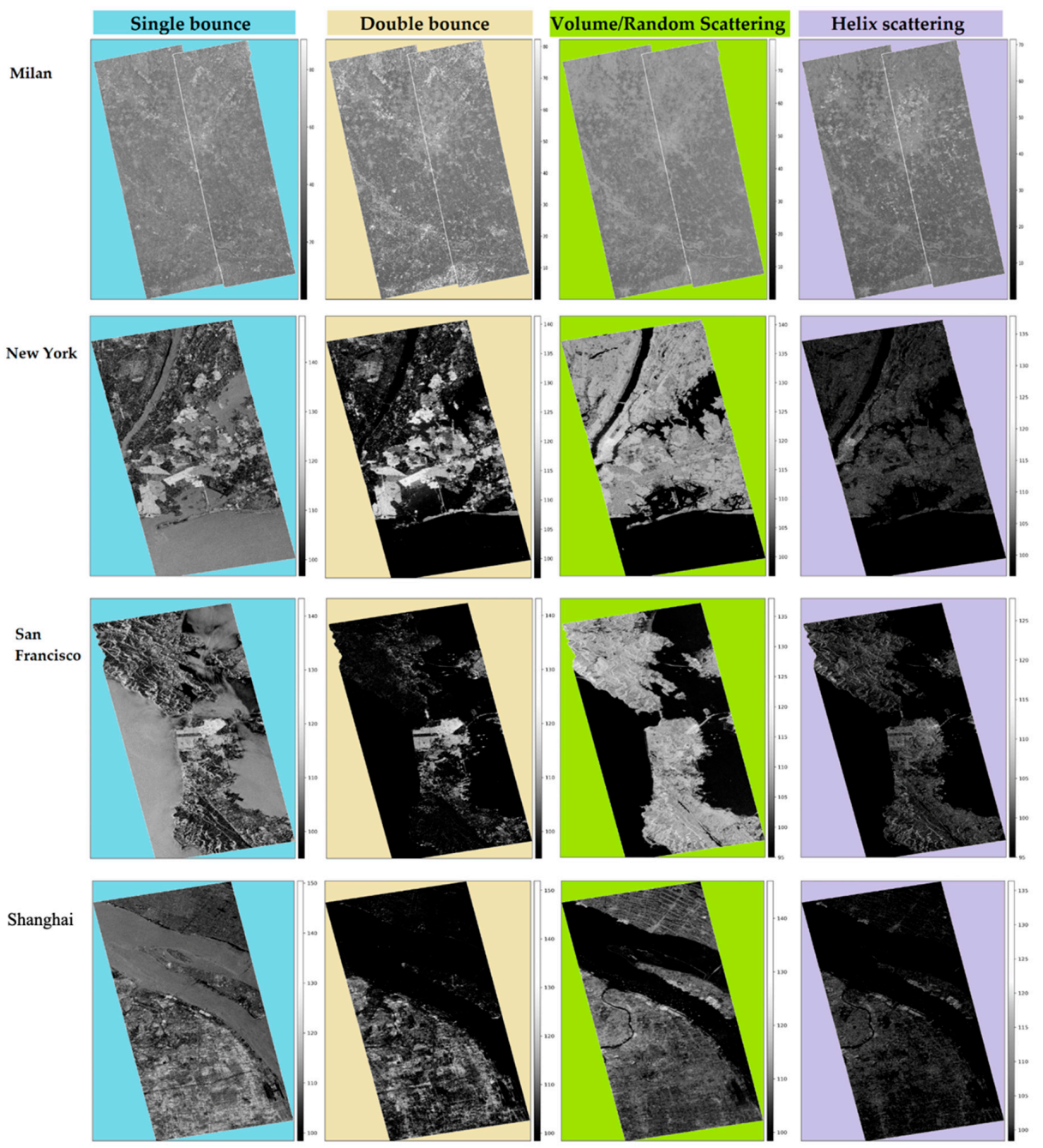

Figure A2. Yamaguchi four-component for Milan, New York, San Francisco and, Shanghai.

\section{References}

1. Lee, J.-S.; Pottier, E. Polarimetric Radar Imaging: From Basics to Applications; CRC Press: Boca Raton, FL, USA, 2009.

2. Patruno, J.; Fitrzyk, M.; Delgado Blasco, J.M. Monitoring and detecting archaeological features with multi-frequency polarimetric analysis. Remote Sens. 2019, 12, 1. [CrossRef]

3. Cloude, S.R.; Member, S.; Pottier, E. An entropy based classification scheme for land applications of polarimetric SAR. Entropy 1997, 35, 68-78. [CrossRef]

4. Freeman, A.; Durden, S.L. A three-component scattering model for polarimetric SAR data. IEEE Trans. Geosci. Remote Sens. 1998, 36, 963-973. [CrossRef]

5. Yamaguchi, Y.; Moriyama, T.; Ishido, M.; Yamada, H. Four-component scattering model for polarimetric SAR image decomposition. IEEE Trans. Geosci. Remote Sens. 2005, 43, 1699-1706. [CrossRef]

6. Atwood, D.K.; Thirion-Lefevre, L. Polarimetric phase and implications for urban classification. IEEE Trans. Geosci. Remote Sens. 2017, 56, 1278-1289. [CrossRef]

7. Yamaguchi, Y.; Yajima, Y.; Yamada, H. A four-component decomposition of POLSAR images based on the coherency matrix. IEEE Geosci. Remote Sens. Lett. 2006, 3. [CrossRef]

8. An, W.; Xie, C.; Yuan, X.; Cui, Y.; Yang, J. Four-component decomposition of polarimetric SAR images with deorientation. IEEE Geosci. Remote Sens. Lett. 2011, 8, 1090-1094. [CrossRef]

9. Pottier, E.; Ferro-Famil, L. PolSARPro V5. 0: An ESA educational toolbox used for self-education in the field of POLSAR and POL-INSAR data analysis. In Proceedings of the 2012 the IEEE International Geoscience and Remote Sensing Symposium, Munich, Germany, 22-27 July 2012; pp. 7377-7380. 
10. Pottier, E.; Lee, J.-S. Application of the $<\mathrm{H} / \mathrm{A} /$ alpha $>$ polarimetric decomposition theorem for unsupervised classification of fully polarimetric SAR data based on the wishart distribution. In Proceedings of the SAR Workshop: CEOS Committee on Earth Observation Satellites, Toulouse, France, 26-29 October 1999; Volume 450, p. 335.

11. Du, L.J.; Lee, J.S. Polarimetric SAR image classification based on target decomposition theorem and complex Wishart distribution. In Proceedings of the IGARSS'96. 1996 International Geoscience and Remote Sensing Symposium, Lincoln, NE, USA, 31 May 1996; Volume 1, pp. 439-441.

12. Tan, C.P.; Lim, K.S.; Ewe, H.T. Image processing in polarimetric SAR images using a hybrid entropy decomposition and maximum likelihood (EDML). In Proceedings of the 2007 5th International Symposium on Image and Signal Processing and Analysis, Istanbul, Turkey, 27-29 September 2007; pp. 418-422.

13. Pottier, E. Radar target decomposition theorems and unsupervized classification of full polarimetric SAR data. In Proceedings of the IGARSS'94-1994 IEEE International Geoscience and Remote Sensing Symposium, Pasadena, CA, USA, 8-12 August 1994; Volume 2, pp. 1139-1141.

14. Fang, C.; Wen, H.; Yirong, W. An improved Cloude-Pottier decomposition using H/ $\alpha /$ span and complex Wishart classifier for polarimetric SAR classification. In Proceedings of the 2006 CIE international conference on radar, Shanghai, China, 16-19 October 2006; pp. 1-4.

15. Ince, T.; Kiranyaz, S.; Gabbouj, M. Evolutionary RBF classifier for polarimetric SAR images. Expert Syst. Appl. 2012, 39, 4710-4717. [CrossRef]

16. Azmedroub, B.; Ouarzeddine, M.; Souissi, B. Extraction of urban areas from polarimetric SAR imagery. IEEE J. Sel. Top. Appl. Earth Obs. Remote Sens. 2016, 9, 2583-2591. [CrossRef]

17. Lee, J.S.; Grunes, M.R.; Ainsworth, T.L.; Du, L.; Schuler, D.L.; Cloude, S.R. Unsupervised classification using polarimetric decomposition and complex Wishart classifier. IEEE Trans. Geosci. Remote Sens. 1999, 37, 2249-2258.

18. Lee, J.-S.; Grunes, M.R.; Kwok, R. Classification of multi-look polarimetric SAR imagery based on complex Wishart distribution. Int. J. Remote Sens. 1994, 15, 2299-2311. [CrossRef]

19. Van Zyl, J.J.; Burnette, C.F. Bayesian classification of polarimetric SAR images using adaptive a priori probabilities. Int. J. Remote Sens. 1992, 13, 835-840. [CrossRef]

20. Loibl, W.; Etminan, G.; Gebetsroither-Geringer, E.; Neumann, H.-M.; Sanchez-Guzman, S. Characteristics of urban agglomeration in different continents: History, patterns, dynamics, drivers and trends, urban agglomeration. In Urban Agglomeration; Ergen, M., Ed.; IntechOpen: London, UK, 2018.

21. Marconcini, M.; Gorelick, N.; Metz-Marconcini, A.; Esch, T. Mapping the global settlement growth from 1985 to 2015-the world settlement footprint evolution dataset. In Proceedings of the AGU Fall Meeting Abstracts, Washington, DC, USA, 10-14 December 2018.

22. Marconcini, M.; Gorelick, N.; Metz-Marconcini, A.; Esch, T. Accurately monitoring urbanization at global scale - the world settlement footprint. In Proceedings of the 20th World Bank Conference on Land and Poverty, Washington, DC, USA, 25-29 March 2019; World Bank: Washington, DC, USA, 2019.

23. Jiang, F.; Liu, S.; Yuan, H.; Zhang, Q. Measuring urban sprawl in Beijing with geo-spatial indices. J. Geogr. Sci. 2007, 17, 469-478. [CrossRef]

24. Chan, C.-S.; Dang, A.; Tong, Z.A.; Tong, Z. A 3D model of the inner city of Beijing. In Computer Aided Architectural Design Futures 2005; Springer: Berlin, Germany, 2005; pp. 63-72.

25. Goldfield, D. Encyclopedia of American Urban History; Sage Publications: Thousand Oaks, CA, USA, 2006.

26. Hassan, A.A.M. Change in the urban spatial structure of the Greater Cairo metropolitan area, Egypt. Archives 2011, XXXVIII, 133-136.

27. Gorelick, N. Google earth engine. In Proceedings of the AGU Fall Meeting Abstracts, San Francisco, CA, USA, 3-7 December 2012; Volume 1, p. 4.

28. Gorelick, N.; Hancher, M.; Dixon, M.; Ilyushchenko, S.; Thau, D.; Moore, R. Google Earth Engine: Planetary-scale geospatial analysis for everyone. Remote Sens. Environ. 2017, 202, 18-27. [CrossRef]

29. Zhang, Y.; Ding, C.; Qiu, X.; Li, F. The characteristics of the multipath scattering and the application for geometry extraction in high-resolution SAR images. IEEE Trans. Geosci. Remote Sens. 2015, 53, 4687-4699.

(C) 2020 by the authors. Licensee MDPI, Basel, Switzerland. This article is an open access article distributed under the terms and conditions of the Creative Commons Attribution (CC BY) license (http://creativecommons.org/licenses/by/4.0/). 